\title{
An inflammatory bowel disease-risk variant in INAVA decreases pattern recognition receptor-induced outcomes
}

\author{
Jie Yan, Matija Hedl, and Clara Abraham \\ Department of Medicine, Yale University, New Haven, Connecticut, USA.
}

\begin{abstract}
Inflammatory bowel disease (IBD) is characterized by dysregulation in both cytokines and responses to intestinal microbes, and proper regulation of pattern recognition receptor (PRR) signaling is critical for intestinal immune homeostasis. Altered functions for the IBD risk locus containing rs7554511, which encompasses the C1orf106 gene (recently named INAVA), and roles for the protein encoded by the INAVA gene are unknown. Here, we investigated the role of INAVA and INAVA genotype in regulating PRR-initiated outcomes in primary human cells. Both peripheral and intestinal myeloid cells expressed INAVA. Upon PRR stimulation, INAVA was required for optimal MAPK and NF-кB activation, cytokine secretion, and intracellular bacterial clearance. INAVA recruited 14-3-3 $\tau$, thereby contributing to recruitment of a signaling complex that amplified downstream signals and cytokines. Further, INAVA enhanced bacterial clearance by regulating reactive oxygen, reactive nitrogen, and autophagy pathways. Macrophages from rs7554511 C risk carriers expressed lower levels of INAVA RNA and protein. Lower expression was attributed in part to decreased transcription mediated directly by the intronic region containing the rs7554511 C variant. In rs7554511 C risk carrier macrophages, lower INAVA expression led to decreased PRR-induced activation of MAPK and NF-KB pathways, cytokines, and bacterial clearance pathways. Thus, IBDassociated polymorphisms in INAVA modulate PRR-initiated signaling, cytokines, and intracellular bacterial clearance, likely contributing to intestinal immune homeostasis.
\end{abstract}

\section{Introduction}

Inflammatory bowel disease (IBD) is characterized by dysregulated intestinal immune homeostasis and cytokine production (1). Microbial recognition and responses are initially modulated by host pattern recognition receptors (PRRs). An important role for host-microbial interactions in human Crohn's disease is particularly highlighted by Crohn's disease-associated loss-of-function polymorphisms in the PRR NOD2 $(1,2)$; these polymorphisms result in decreased signaling and cytokine secretion in response to NOD2 stimulation. In intestinal tissues, multiple other PRRs are activated, the outcomes of which might in turn be modulated by additional IBD risk loci. Polymorphisms resulting in both decreased (e.g., NOD2, IL18RAP, ICOSL, ATG16L1, CARD9) and increased (e.g., MAP3K8, TNFSF15, IRF5) PRR-mediated signaling and downstream outcomes can be associated with intestinal inflammation (2-11), thereby highlighting the critical role of balance in regulation of PRR-initiated outcomes in intestinal tissues. Despite the success in identification of IBD-associated loci (12), altered functions for most of the IBD loci are unknown. One such region is on chromosome 1, encompassing the C1orf106 gene (now named INAVA [innate immune activator]); the kinesin family member 21B (KIF21B) gene in this region is one of the genes that has been hypothesized to account for the IBD risk association (12). The IBD rs7554511 C risk variant is located in an intronic region between exons 6 and 7 of INAVA (12); it is a common variant observed at

Conflict of interest: The authors have declared that no conflict of interest exists. Submitted: January 4, 2016; Accepted: February 16, 2017.

Reference information: / Clin Invest. 2017;127(6):2192-2205.

https://doi.org/10.1172/JCI86282 a 0.641-0.726 frequency in European ancestry healthy individuals (per dbSNP; https://www.ncbi.nlm.nih.gov/projects/SNP/), such that it likely represents the ancestral allele. The rs7554511 C risk allele confers a 1.10-1.164 increased risk of developing IBD (12-14) (1.153 and 1.176 for Crohn's disease and ulcerative colitis, respectively; see ref. 12). Another variant in this gene region (rs12122721) is associated with multiple sclerosis (15). Functions for the protein encoded by INAVA have not yet been reported. However, given that polymorphisms in the region encompassing this gene are associated with IBD, we questioned if INAVA regulates PRR-initiated outcomes, and if so, the mechanism(s) through which this regulation occurs. We further hypothesized that these outcomes would be modulated by INAVA genotype.

In this study, we focused on contributions and mechanisms for INAVA-mediated regulation of PRR-initiated outcomes in primary human cells, given the association of the INAVA gene with human disease and the dramatic differences that can be observed between human and mouse immune-mediated pathways (16). INAVA was expressed in both peripheral and intestinal myeloidderived cells, and was required for optimal PRR-induced signaling, cytokine secretion, and bacterial clearance in primary human monocyte-derived macrophages (MDMs). INAVA associated with 14-3-3 $\tau$, which in turn led to the recruitment of a signaling complex that amplified PRR-induced downstream signals and cytokine secretion. Importantly, MDMs from rs7554511 C risk carriers expressed lower levels of INAVA, and demonstrated decreased signaling, cytokine secretion, and bacterial clearance upon stimulation with ligands for a broad range of PRRs. Taken together, these results identify a role for a previously undefined protein encoded by INAVA and establish clear roles and mechanisms for 

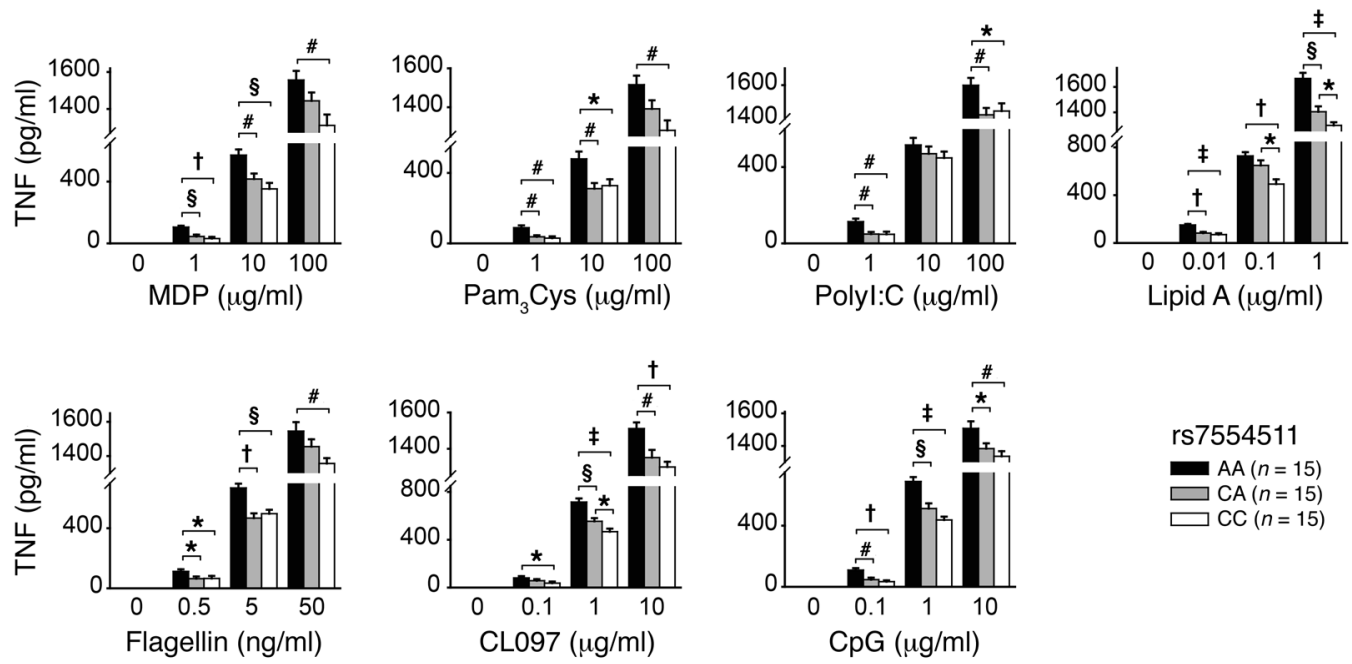

Figure 1. Human myeloid cells from rs7554511 C risk allele carriers demonstrate less cytokine secretion upon PRR stimulation compared with AA carriers. Human MDMs ( $n=15$ /genotype) were treated for 24 hours with the indicated doses of MDP (recognized by NOD2), Pam ${ }_{3}$ Cys (recognized by TLR2), polyl:C (recognized by TLR3), lipid A (recognized by TLR4), flagellin (recognized by TLR5), CL097 (recognized by TLR7), or CpC DNA (recognized by TLR9). Shown is TNF secretion. ${ }^{*} P<0.05$; ${ }^{\sharp} P<0.01 ;{ }^{\circledR} P<0.001 ;{ }^{\dagger} P<1 \times 10^{-4} ;{ }^{\ddagger} P<1 \times 10^{-5}$; determined by 2 -tailed Student's $t$ test.

the protein in regulating PRR-initiated outcomes, as well as establishing loss-of-function consequences for the IBD-associated risk variant in this gene.

\section{Results}

MDMs from rs 7554511 C risk carriers in INAVA demonstrate decreased PRR-induced secretion of cytokines. PRR-initiated outcomes, including cytokine secretion, in myeloid-derived cells are important in IBD pathophysiology (2). As the rs7554511 polymorphism in INAVA is associated with IBD, we questioned if PRRinitiated cytokine secretion from primary human monocytederived cells is modulated by INAVA genotype. Given the association of NOD2 with Crohn's disease (1), we initially utilized muramyl dipeptide (MDP), the component of peptidoglycan that specifically activates NOD2 (17-20), to treat MDMs. We examined TNF secretion, given its role in IBD (1). MDMs from rs7554511 $C$ risk carriers secreted less TNF upon NOD2 stimulation compared with AA carriers across a range of MDP doses (Figure 1). We observed similar regulation of yet another proinflammatory cytokine, IL-1 $\beta$ (Supplemental Figure 1A; supplemental material available online with this article; https://doi.org/10.1172/ JCI86282DS1). Similar results were observed with the antiinflammatory cytokine IL-10 (Supplemental Figure 1B), such that the polymorphism regulates both pro- and antiinflammatory cytokines. Microbial products activate multiple PRRs. Upon dosedependent stimulation of multiple TLRs, relative to AA carriers, MDMs from rs7554511 C risk carriers secreted less TNF, IL-1 $\beta$, and IL-10 (Figure 1 and Supplemental Figure 1). We further stratified on multiple polymorphisms within the rs7554511 region and found that the rs7554511 variant (along with those variants in linkage disequilibrium [LD] with rs7554511) was associated with the most significant modulation of NOD2-induced cytokines (Supplemental Figure 2). Therefore, relative to rs7554511 AA carriers, MDMs from $\mathrm{C}$ risk carriers exhibit lower cytokine secretion upon stimulation of a broad range of PRRs.
INAVA is required for optimal PRR-induced cytokine secretion from MDMs. Rs7554511 is located in an intronic region of the INAVA gene. However, given the lack of prior reports on this gene, and the presence of other genes in the region (Supplemental Figure 3A), we considered that any one or all of the genes may be accounting for the genotype-dependent effects observed. We therefore first effectively knocked down each of the genes within a 500-kb distance from rs7554511 in human MDMs (Supplemental Figure $3 \mathrm{~B}$ ), and examined which of the candidate genes regulated NOD2-induced cytokines. INAVA knockdown led to a significant decrease in NOD2-induced proinflammatory and antiinflammatory cytokines (Figure 2A), whereas knockdown of the other genes in the region did not (Supplemental Figure 3C). Knocking down INAVA in MDMs did not affect cell viability (Supplemental Figure 4A), and cells remained responsive to alternative stimuli to the dectin receptor (Supplemental Figure 4B), a receptor that responds to fungal products and initiates distinct proximal signaling pathways (21). INAVA regulated NOD2 responses over a wide range of MDP doses (Supplemental Figure 4C). The differential effect with INAVA knockdown was most visible at the highest MDP dose (Supplemental Figure 4C), such that we will use this dose in the studies that ensue. NOD2-induced cytokines were decreased upon INAVA knockdown with 3 additional siRNAs (Supplemental Figure 4, D and E). Furthermore, knockdown of INAVA led to decreased cytokine secretion upon stimulation of a broad range of PRRs (Figure 2B). Consistently, INAVA regulated both MyD88dependent and -independent outcomes upon stimulation of TLR4 (Supplemental Figure 5). Therefore, INAVA is required for optimal responses upon stimulation of a broad range of PRRs.

INAVA expression is induced in human MDMs with PRR stimulation and INAVA is highly expressed in intestinal myeloid-derived cells. Given the intronic location of rs7554511, we considered that INAVA expression might be modulated in an rs7554511 genotypespecific manner. We therefore first examined if INAVA expression was induced with NOD2 stimulation. Expression of INAVA mRNA 


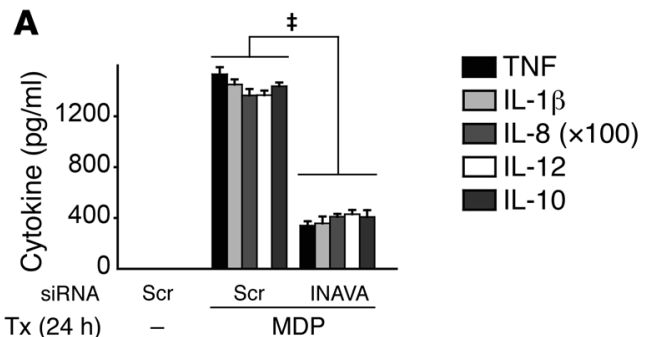

B
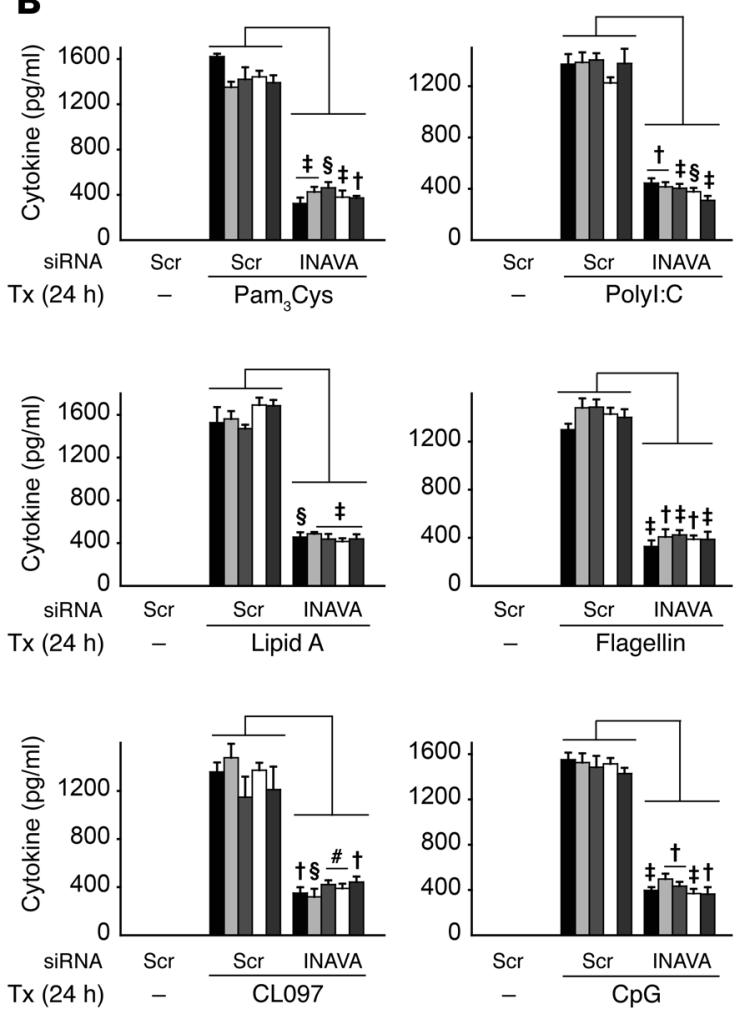

peaked 8 hours after NOD2 stimulation (Figure 3A). There are 2 INAVA transcripts (NM_018265.3 and NM_001142569.2) identified to date that encode for proteins; both transcripts revealed similar regulation upon NOD2 stimulation (Supplemental Figure 6A). Further, upon INAVA knockdown, both transcripts were effectively reduced (Supplemental Figure 6B). INAVA protein expression also increased with NOD2 stimulation as assessed by intracellular flow cytometry (Figure 3B). We ensured specificity of the antibody through siRNA to INAVA as assessed by protein expression through 2 independent approaches (Figure 3C and Supplemental Figure 6C). Increased INAVA protein expression upon NOD2 stimulation was also observed by Western blot (Figure 3D). We further confirmed the induction of INAVA protein with a second anti-INAVA (C1orf106) antibody by intracellular flow cytometry (Supplemental Figure 6D), with specificity confirmed by knockdown of INAVA (Supplemental Figure 6E). Given the association of rs7554511 in INAVA with IBD, a disease of intestinal immune dysregulation, we also examined if INAVA was expressed in human intestinal myeloid-derived cells, and found that INAVA mRNA was higher relative to its expression in MDMs (Figure 3E). This is consistent with the PRR ligand-induced expression of INAVA (Figure 3, A
Figure 2. INAVA is required for optimal secretion of cytokines in MDMs upon stimulation through a broad range of PRRs. Human MDMs $(n=4)$ were transfected with scrambled or INAVA siRNA. Cells were treated with (A) $100 \mu \mathrm{g} / \mathrm{ml}$ MDP or (B) $10 \mu \mathrm{g} / \mathrm{ml} \mathrm{Pam}_{3}$ Cys (recognized by TLR2), $100 \mu \mathrm{g} /$ $\mathrm{ml}$ polyl:C (recognized by TLR3), $0.1 \mu \mathrm{g} / \mathrm{ml}$ lipid A (recognized by TLR4), 5 $\mathrm{ng} / \mathrm{ml}$ flagellin (recognized by TLR5), $1 \mu \mathrm{g} / \mathrm{ml}$ CL097 (recognized by TLR7), or $10 \mu \mathrm{g} / \mathrm{ml}$ CpC DNA (recognized by TLR9) for 24 hours. Mean cytokine secretion + SEM is shown. Similar results were observed in an additional $n=16$ for $\mathbf{A}$ and $n=8$ for $\mathbf{B}$. Tx, treatment; Scr, scrambled. ${ }^{P} P<0.01$; ${ }^{\circledR} P<$ $0.001 ;{ }^{\dagger} P<1 \times 10^{-4} ;{ }^{\ddagger} P<1 \times 10^{-5}$; determined by 2-tailed Student's $t$ test.

and $\mathrm{B}$ ) and with cells in the intestinal lamina propria being continuously exposed to microbial ligands $(22,23)$.

MDMs from rs $7554511 \mathrm{C}$ risk carriers demonstrate lower INAVA expression. We next questioned if INAVA expression was regulated in an rs7554511 genotype-dependent manner. Consistent with the lower cytokine secretion from MDMs of rs7554511 CC risk carriers, MDMs from rs7554511 CC carriers expressed less INAVA mRNA than AA carriers at baseline; this differential was further enhanced upon NOD2 stimulation (Figure 4A) and was observed in both INAVA transcripts (Figure 4A). The ratio of the transcripts was not significantly different with rs7554511 genotype (data not shown). MDMs from rs7554511 heterozygote carriers generally demonstrated an intermediate level of INAVA mRNA expression (Figure 4A). Lower INAVA expression was also observed at the protein level in rs7554511 CC risk carriers (Figure 4B). Of note, for the genes in the region that did not regulate NOD2-induced cytokines as per Supplemental Figure 3C, their expression was also not modulated in a genotype-dependent manner in MDMs (Supplemental Figure 7). Therefore, INAVA expression increases with NOD2 stimulation, and MDMs from rs7554511 C risk carriers express less INAVA relative to AA carriers.

Modulation in INAVA expression levels by the rs 7554511 variant accounts for the INAVA-dependent PRR-induced cytokine secretion. We sought to clearly establish that modulation in INAVA expression levels accounted for the INAVA genotype-dependent regulation of PRR-induced cytokines. Therefore, we progressively reduced the levels of INAVA in MDMs from high-expressing rs7554511 AA carriers to the levels observed in CC carriers, as well as beyond these levels in accordance with the degree of protein reduction in the knockdown studies above (Supplemental Figure 8A). With reduction of INAVA expression in AA carrier MDMs to the levels observed in CC carrier MDMs, cells demonstrated similar levels of NOD2-induced cytokine secretion compared with rs7554511 CC carrier MDMs (Supplemental Figure 8B). Interestingly, in examining the relationship between INAVA expression and NOD2induced cytokines over a broader range of INAVA concentrations, there was a clear threshold of INAVA expression at which NOD2induced cytokines more rapidly decreased (Supplemental Figure 8C). We then conducted the complementary studies wherein we increased INAVA expression in rs7554511 CC risk-carrier MDMs to the levels observed in rs7554511 AA carrier MDMs (Supplemental Figure 9A); this resulted in similar levels of cytokine secretion compared with AA carrier MDMs (Supplemental Figure 9B). Therefore, the modulation in INAVA expression levels associated with the intronic rs7554511 variant in the INAVA region accounts for the regulation in the INAVA-dependent outcomes observed. 


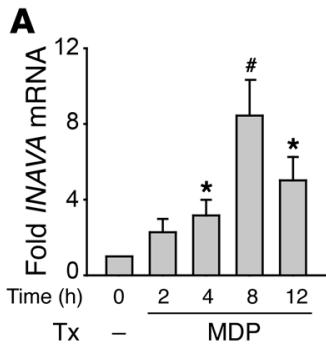

\section{B}
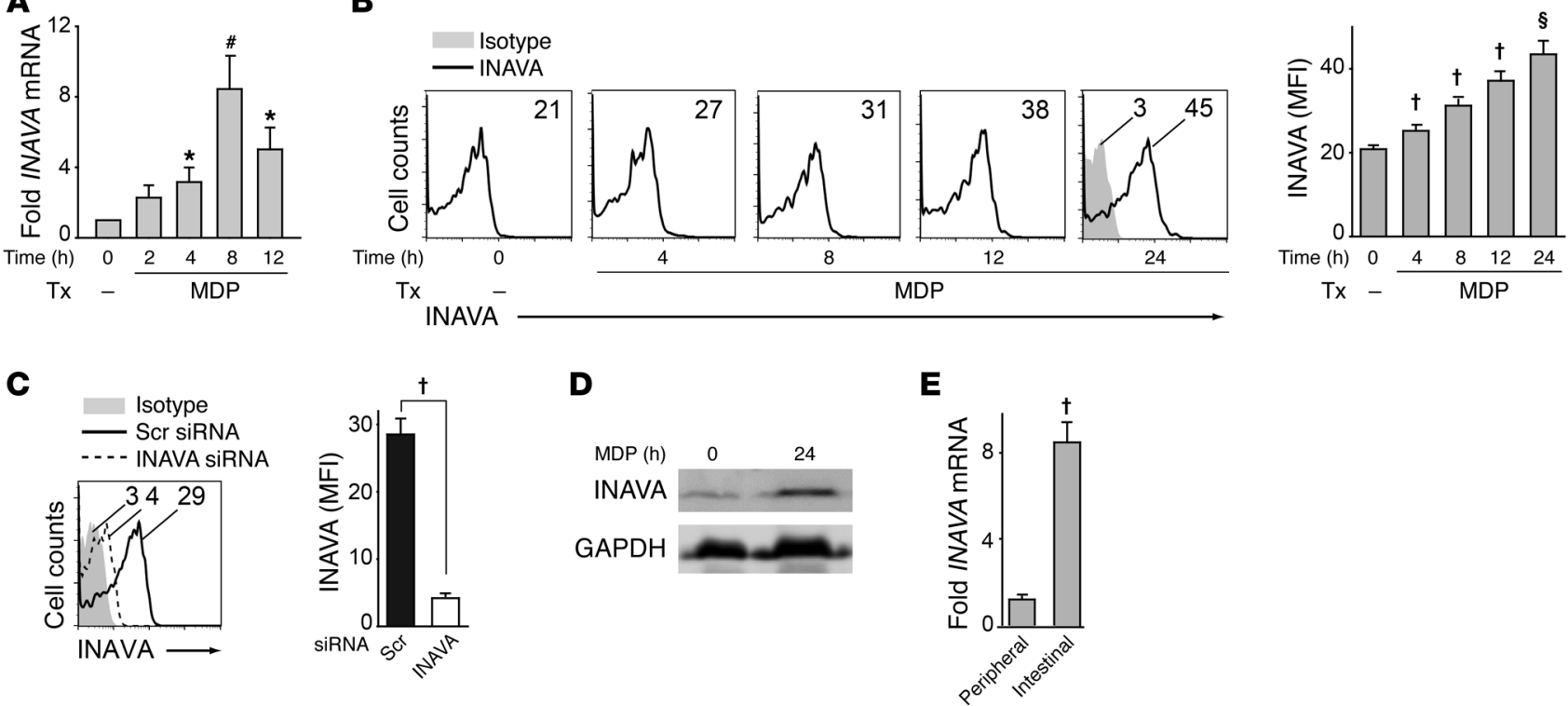

Figure 3. INAVA is expressed in peripheral and intestinal myeloid-derived cells, and INAVA expression increases with PRR stimulation. (A) Human MDMs $(n=10)$ were stimulated for the indicated times with $100 \mu \mathrm{g} / \mathrm{ml} \mathrm{MDP.} \mathrm{INAVA} \mathrm{mRNA} \mathrm{expression} \mathrm{was} \mathrm{normalized} \mathrm{to} \mathrm{untreated} \mathrm{MDMs} \mathrm{and} \mathrm{GAPDH.}$ Mean + SEM is shown. GAPDH expression was stable at each of these time points and similar INAVA mRNA regulation was observed with normalization to a different housekeeping gene, ACTB (data not shown). (B) MDMs $(n=8)$ were treated with $100 \mu \mathrm{g} / \mathrm{ml} \mathrm{MDP}$ for the indicated times and INAVA protein levels were assessed by flow cytometry. Left: Representative flow cytometry plots with INAVA mean fluorescence intensity (MFI) values. Right: Summary of INAVA protein expression + SEM. Similar results were observed in an additional $n=8$. (C) MDMs were transfected with scrambled or INAVA siRNA and assessed for INAVA protein expression. Representative flow cytometry plot with summary graph $(n=8)$ for MFI + SEM are shown. (D) MDMs were treated with $100 \mu \mathrm{g} / \mathrm{ml}$ MDP for 24 hours and assessed for INAVA protein expression by Western blot (representative for 1 of 7 individuals). GAPDH was used as a loading control; GAPDH levels were stable over the time course examined (data not shown). (E) INAVA mRNA expression was assessed in intestinal ( $n=7$ ) and peripheral $(n=7)$ myeloid-derived cells and normalized to CD11c. Mean + SEM. Tx, treatment; scr, scrambled. ${ }^{*} P<0.05 ;{ }^{\sharp} P<0.01 ;{ }^{\circledR} P<0.001 ;$ ${ }^{\dagger} P<1 \times 10^{-4}$; determined by 2 -tailed Student's $t$ test.

The INAVA rs7554511 variant alters intron-driven transcription. As INAVA expression modulation accounted for the rs7554511 genotype-dependent effects on PRR-induced cytokines, we next sought to understand the mechanisms regulating INAVA expression, and which of these mechanisms might account for the rs7554511-modulated INAVA expression regulation. Gene expression can be dramatically modulated by miRNA binding, and there is a putative miRNA-24 binding site in the $3^{\prime}$ UTR of INAVA (Supplemental Figure 10A). Upon miRNA-24 mimic overexpression in MDMs, INAVA expression levels decreased, while with a miRNA-24 hairpin inhibitor, INAVA expression levels increased (Supplemental Figure 10B). Consistent with INAVA expression levels regulating NOD2-induced cytokines, miRNA-24 mimic overexpression reduced NOD2-induced cytokines, while the miRNA-24 hairpin inhibitor increased NOD2-induced cytokines (Supplemental Figure 10C). To more clearly examine the putative region within the $3^{\prime}$ UTR of INAVA containing miRNA-24, and to determine if there were genetic variants regulating miRNA-24modulated INAVA expression, we subcloned a portion of the $3^{\prime}$ UTR region of INAVA containing the predicted miRNA-24 binding site into a luciferase construct driven by a $P G K$ promoter. When this INAVA 3' UTR miRNA-24 region construct was expressed in HEK293 cells along with miRNA-24, luciferase expression from the INAVA 3' UTR was reduced, whereas with the miRNA-24 hairpin inhibitor, luciferase expression was increased (Supplemental Figure 10D). Luciferase activity in a construct in which the con- sensus nucleotides of the miRNA-24 binding site in INAVA were mutated (as per Supplemental Figure 10A) was not regulated with miRNA-24 mimic or hairpin inhibitor (Supplemental Figure 10D), thereby establishing the specificity of the miRNA-24 regulation. Per dbSNP there are 2 single nucleotide polymorphisms (SNPs) at rs1048978 and rs558221123 contained within the miRNA-24 binding region. Mutating these sites to the respective derived variant did not alter luciferase regulation relative to the ancestral variant (Supplemental Figure 10D). Mutating a third SNP (rs35084944) described in dbSNP just outside of the predicted miRNA-24 region also did not alter the pattern of luciferase regulation from the INAVA 3' UTR gene region examined. Furthermore, these SNPs were not in LD with the rs7554511 polymorphism. Therefore, while miRNA-24 binding in the 3' UTR of INAVA decreases INAVA expression, the identified SNPs in this region neither modulate this regulation nor are in LD with the INAVA IBD-associated rs7554511 variant.

We next questioned if the intronic region where rs7554511 is located regulates INAVA expression, and if so, if this expression is regulated in a rs7554511 genotype-dependent manner. We identified various transcription factor consensus sites that directly overlap with the rs7554511 variant in intron 6 (Supplemental Figure 11A). Through knockdown of endogenous transcription factors in MDMs (Supplemental Figure 11B), we identified that TATA boxbinding protein (TBP) and homeobox A5 (HOXA5) were required for both optimal baseline and NOD2-induced INAVA RNA expres- 

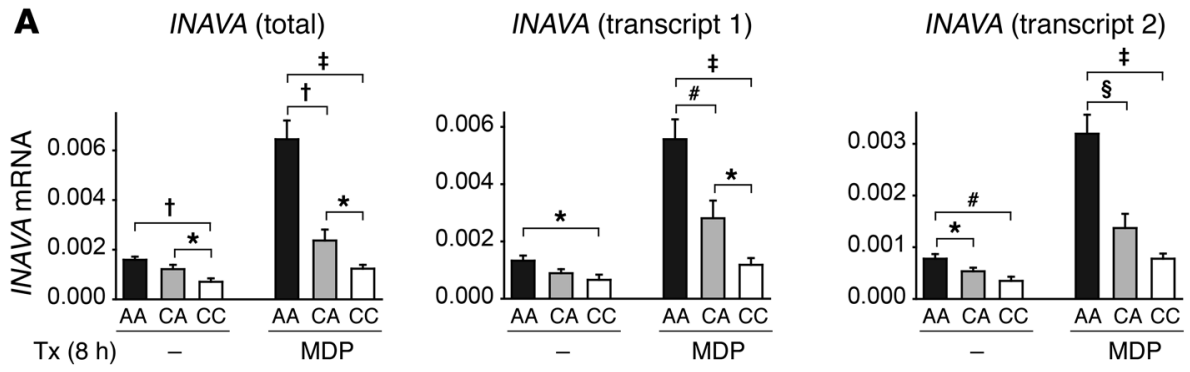

B
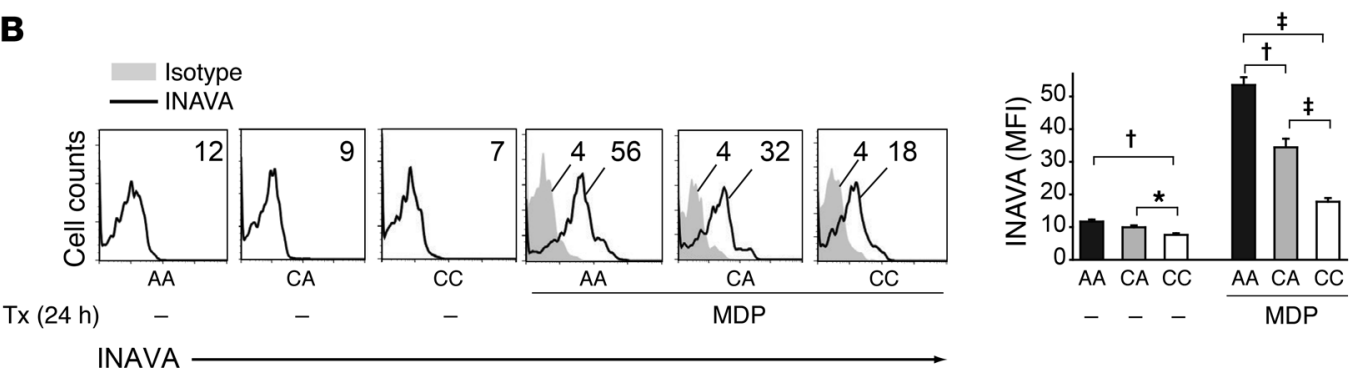

Figure 4. MDMs from rs7554511 C risk carriers express less INAVA. (A and B) MDMs from rs7554511 AA, CA, and CC carriers ( $n=15 /$ genotype, similar results were seen in an additional $n=8$ /genotype) were left untreated or treated with $100 \mu \mathrm{g} / \mathrm{ml}$ MDP for 8 hours (A) or 24 hours (B). (A) INAVA mRNA expression (expressed as change in Ct values normalized to GAPDH and represented as a linear scale) + SEM. (B) INAVA protein expression with (left) representative flow cytometry and mean fluorescence intensity (MFI) values shown, and (right) summarized data for mean $+\mathrm{SEM}$. Tx, treatment. ${ }^{*} P<0.05$; ${ }^{\#} P<0.01 ;{ }^{\circledR} P<0.001 ;{ }^{\dagger} P<1 \times 10^{-4} ;{ }^{\ddagger} P<1 \times 10^{-5}$; determined by 2-tailed Student's $t$ test.

sion, whereas NKX2-5 was not (Supplemental Figure 11C). As TBP contributed to a greater degree to NOD2-induced INAVA RNA expression, we focused on TBP and included NKX2-5 as a negative control. Similar to mRNA regulation, TBP regulated baseline and NOD2-induced INAVA protein expression in MDMs (Supplemental Figure 11D). Consistent with these findings, TBP was required for NOD2-induced cytokines, whereas NKX2-5 was not (Supplemental Figure 11E).

As TBP might be regulating INAVA expression through locations in INAVA in addition to the putative binding site in intron 6, we subcloned intron 6 (842 bp) containing the rs7554511 A variant upstream of a luciferase reporter construct to determine potential regulation by TBP specifically in the intron 6 region. Upon transfection of this construct into HEK293 cells, we observed INAVA intron 6-driven luciferase expression and this expression was increased with MDP treatment (Figure 5A). Through knockdown of TBP (Supplemental Figure 11F), we found that TBP was required for optimal INAVA intron 6-driven luciferase expression, both at baseline and upon MDP treatment (Figure 5A). Given the decreased INAVA expression in MDMs from rs7554511 C risk carriers, we next questioned if intron 6 containing the rs7554511 C risk variant demonstrated less transcriptional activity than did the rs7554511 A variant, and we found this to be the case (Figure 5B). Importantly, expressing TBP in HEK293 cells transfected with the intron 6 rs7554511 A variant significantly enhanced NOD2induced transcription (Figure $5 \mathrm{C}$ ). The transcriptional activity in the presence of TBP was less in the intron 6 rs7554511 C variant relative to the A variant (Figure $5 \mathrm{C}$ ). A specificity control in which a subset of the base pairs within the TBP consensus binding sequence were mutated (Supplemental Figure 11A) demonstrated a failure to upregulate transcriptional activity with TBP expression
(Figure $5 \mathrm{C}$ ). Taken together, these results demonstrate that the intron 6 rs7554511 A variant has increased transcriptional activity relative to the $\mathrm{C}$ variant, with TBP being one transcription factor mediating the increased transcriptional activity.

MDMs from rs7554511 C risk carriers in INAVA demonstrate decreased PRR-induced MAPK and NF- $\kappa B$ activation. We next questioned the mechanisms through which INAVA regulates PRRinitiated cytokines. We first examined the signaling pathways regulated by INAVA upon NOD2 stimulation. Activation of MAPK and $\mathrm{NF}-\kappa \mathrm{B}$ pathways is critical for NOD2-induced cytokines $(6,7,19$, 24-26). Activation of the MAPKs ERK, p38, and JNK (Figure 6A), and the NF- $\kappa$ B pathway (Figure $6 \mathrm{~B}$ ) were impaired upon NOD2 stimulation of MDMs in which INAVA was knocked down. Consistently, binding of transcription factors to cytokine promoters downstream of these pathways, including c-Jun, c-Fos, and NF-kBp65, was decreased upon INAVA knockdown in MDMs with NOD2 stimulation (Supplemental Figure 12). Moreover, signaling downstream of multiple PRRs was decreased with INAVA knockdown (Supplemental Figure 13). Finally, we questioned if the INAVA-dependent signaling pathways observed with NOD2 stimulation were regulated in an rs7554511 genotype-dependent manner; we focused on rs7554511 AA and CC carriers for these studies. Consistent with the lower INAVA expression (Figure 4), and decreased cytokine secretion (Figure 1), NOD2-induced activation of the MAPK (Figure 6C) and NF- $\kappa \mathrm{B}$ (Figure 6D) pathways was decreased in MDMs from rs7554511 CC carriers relative to AA carriers.

INAVA translocates to the nucleus upon NOD2 stimulation. To further define mechanisms for INAVA contributions to PRRinduced signaling, we considered the structural regions of INAVA that might, in turn, be regulating functional outcomes. We noted that there were 3 potential nuclear localization signals (NLSs) in 
A

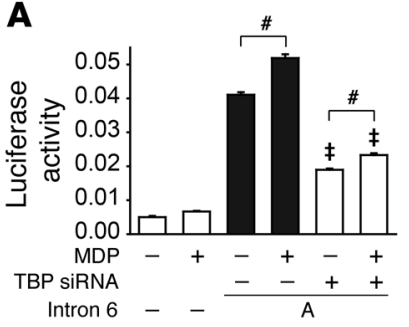

B

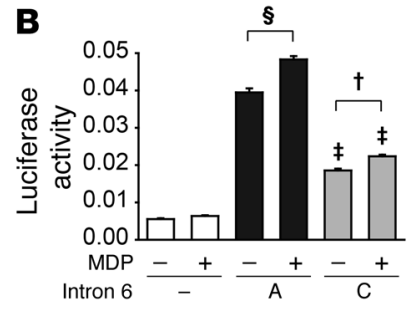

C

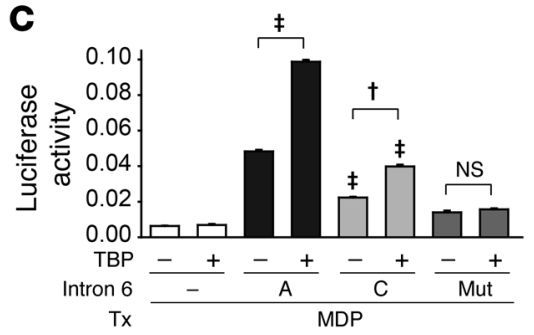

Figure 5. The INAVA rs7554511 C risk variant results in lower intron 6-mediated transcription and lower TBP-mediated transcription within intron 6. (A-C) INAVA intron 6 (842 bp) expressing the indicated rs7554511 variants or the control pGL4.17 vector were transfected into HEK293 cells along with Renilla and NOD2 vector. Cells were additionally transfected with (A) scrambled or TBP siRNA, or (C) empty vector or TBP vector. Cells were then treated with $100 \mu \mathrm{g} / \mathrm{ml}$ MDP for 24 hours and luciferase activity was assessed in 6 replicates. The INAVA intron 6 TCCCCT mutant (mut) (see Supplemental Figure 11A) served as a specificity control for the transcription factor TBP. Representative of 3 independent experiments. Significance is shown for (A) scrambled compared with TBP siRNA for the same conditions in the intron 6-transfected cells or (B and C) rs7554511 C compared with A variants for the same conditions or as indicated. Tx, treatment; NS, not significant. ${ }^{\sharp} P<0.01 ;{ }^{\circledR} P<0.001 ;{ }^{\dagger} P<1 \times 10^{-4}$; ${ }^{\ddagger} P<1 \times 10^{-5}$; determined by 2 -tailed Student's $t$ test.

INAVA (Supplemental Figure 14A). We therefore first questioned if INAVA translocated to the nucleus upon NOD2 stimulation. We observed peak INAVA nuclear translocation 2 hours after MDP treatment of MDMs (Supplemental Figure 14B). To establish if the putative NLSs contributed to the nuclear translocation, and in turn, NOD2-induced signaling and cytokines, we generated INAVA mutants in which each of the 3 putative NLSs was mutated alone (mNLS), and in combination, and transfected these into HEK293 cells along with NOD2. Translocation of INAVA was only slightly decreased with transfection of INAVA mNLS1, mNLS2, or mNLS3 relative to WT INAVA upon NOD2 stimulation (Supplemental Figure 14C). NOD2-induced AP-1 and NF- $\kappa$ B activation (Supplemental Figure 14D) and IL-6 secretion (Supplemental Figure 14E) were not significantly altered with transfection of INAVA mNLS1, mNLS2, or mNLS3 relative to WT INAVA. However, mutation of all 3 NLSs in combination resulted in a failure of INAVA nuclear translocation upon NOD2 stimulation (Supplemental Figure $14 \mathrm{C}$ ), and this was accompanied by a modest, albeit significant reduction in NOD2-induced AP-1 and NF- $\mathrm{BB}$ activation and IL-6 secretion (Supplemental Figure 14, D and E). We did not observe increased INAVA binding to cytokine promoters in MDMs upon NOD2 stimulation as assessed by ChIP (Supplemental Figure $14 \mathrm{~F})$. Therefore, although INAVA translocates to the nucleus upon NOD2 stimulation and this translocation requires the cooperation of 3 NLSs in INAVA, this nuclear translocation event contributes to NOD2-induced signaling and cytokines only to a minor degree.

INAVA associates with 14-3-3 $\tau$ and additional signaling molecules upon PRR stimulation. To further define the mechanisms through which INAVA regulates NOD2- and PRR-initiated signaling, we next considered cytoplasm-associated contributions for INAVA and questioned if INAVA could directly associate with NOD2 and RIP2, the adaptor molecule required for NOD2-initiated signaling. We found that this was the case (Figure 7A). We also found that IRAK1, which is required for proximal NOD2/RIP2-initiated signaling $(20,27)$, associated in a complex with INAVA (Figure 7A). We confirmed that NOD2, RIP2, and IRAK1 were required for the MDP-induced signaling (Supplemental Figure 15A) and cytokine secretion (Supplemental Figure 15B) observed in MDMs. We next considered additional structural regions of INAVA and how they, in turn, might regulate the ability of INAVA to assemble a signal- ing complex. We identified 3 putative 14-3-3 binding domains in INAVA (Figure 7B); 14-3-3 proteins can serve as scaffolding proteins that then recruit a diverse array of signaling proteins (28). While 14-3-3 proteins have been well described in regulating a variety of cell processes, including cell cycle progression and cancer $(29,30)$, relatively few studies have dissected their role in modulating signaling downstream of PRRs (31-34). The putative 14-3-3 binding domains in INAVA, in particular the serine residues mediating binding, are conserved across species (Figure 7B). In fact, the full INAVA protein has an identity ranging from $99.7 \%$ in mammals to $37 \%$ in zebrafish (Supplemental Figure 16A). Moreover, in examining a phylogenetic tree, the INAVA (C1orf106) gene family arose early in bony fish evolution; the genes encoding FRMD4A, FRMD4B, and CCDC120 are potential paralogs of INAVA (Supplemental Figure 16B). Given the identified 14-3-3 binding regions in INAVA, we first examined if 14-3-3 is recruited to INAVA. We focused on 14-3-3 $\tau$ (also known as YWHAQ or 14-3-3 protein theta), given a report suggesting a role for this member in enhancing select measures downstream of TLR activation (34). We observed that there was a baseline association of 14-3-3 $\tau$ with INAVA under unstimulated conditions, and this association increased within 15 minutes of NOD2 stimulation in MDMs (Figure 7C). Of note is that $14-3-3 \tau$ protein expression did not increase with NOD2 stimulation (Supplemental Figure 17A). 14-3-3 can recruit various signaling molecules, including activated MAPKs and NF- $\kappa \mathrm{B}(28$, 35), pathways we had observed to be modulated by INAVA upon PRR stimulation (Figure 6). We therefore examined if p-ERK, $\mathrm{p}-\mathrm{p} 38$, and $\mathrm{p}-\mathrm{I} \kappa \mathrm{B} \alpha$ were recruited to INAVA upon NOD2 stimulation of MDMs, and we found this to be the case (Figure 7C). There was a baseline association of unphosphorylated ERK and p38 in a complex with INAVA, and the recruitment of these signaling molecules increased with NOD2 stimulation (Figure 7C). To further establish the role of 14-3-3 $\tau$ in INAVA modulation of NOD2 signaling, we effectively knocked down 14-3-3 $\tau$ (Supplemental Figure $17, \mathrm{~B}$ and $\mathrm{C})$. We verified that $14-3-3 \tau$ recruitment to INAVA was significantly attenuated under these conditions (Figure 7D). We then examined $\mathrm{p}$-ERK recruitment as one of the signaling proteins we had identified to be recruited to INAVA. Recruitment of p-ERK to INAVA upon NOD2 stimulation was impaired upon 14-3-3 $\tau$ knockdown in MDMs (Figure 7D), thereby confirming the role of 


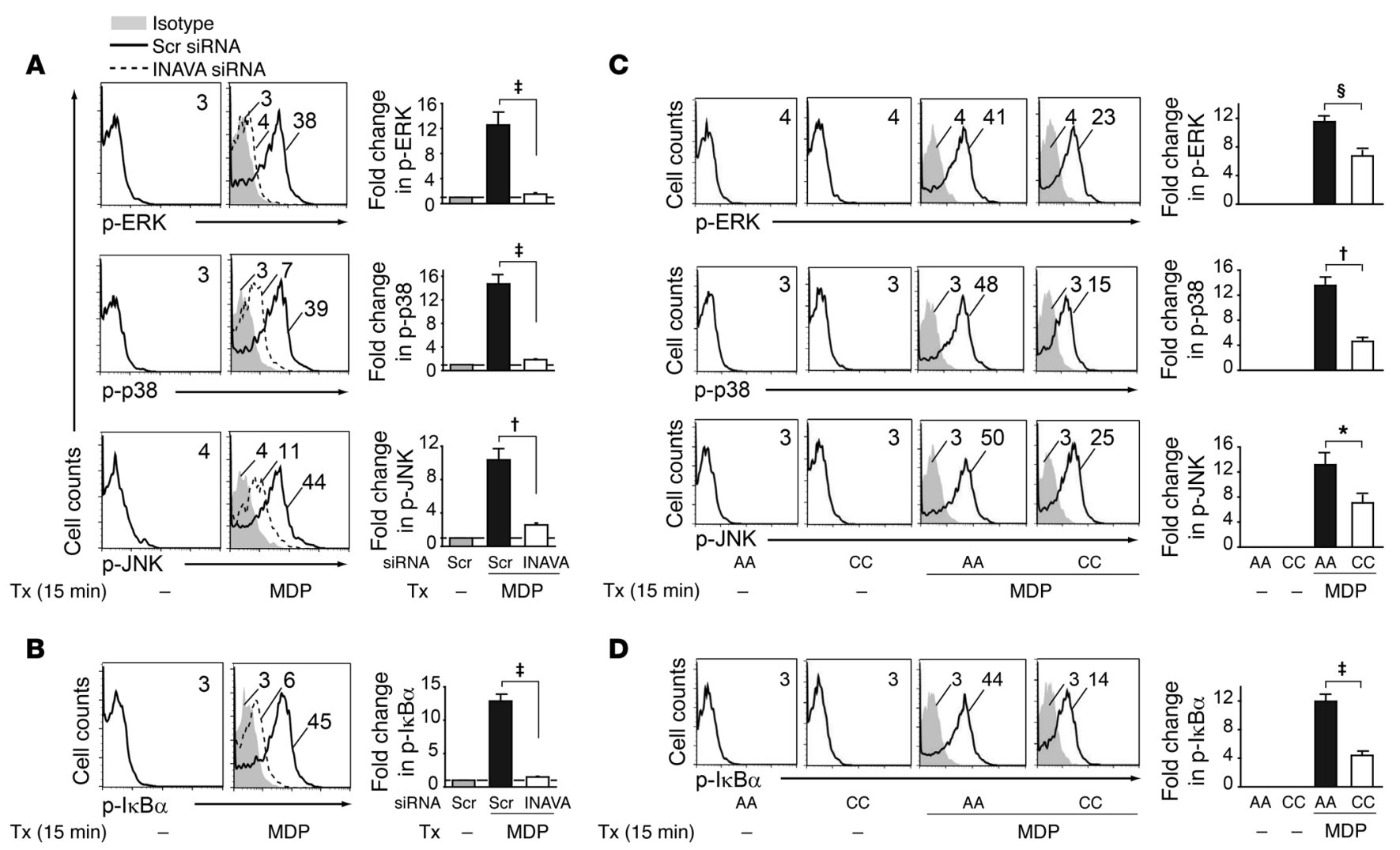

Figure 6. INAVA is required for optimal NOD2-initiated MAPK and NF-кB signaling in MDMs and is decreased in MDMs from INAVA rs7554511 C risk carriers. (A and B) MDMs $(n=8)$ were transfected with scrambled or INAVA siRNA. Cells were then treated with $100 \mu \mathrm{g} / \mathrm{ml}$ MDP for 15 minutes and assessed for (A) p-ERK, p-p38, p-JNK, or (B) p-IKB $\alpha$. Left: Representative flow cytometry plots with mean fluorescence intensity (MFI) values. Right: Fold phosphoprotein induction normalized to untreated, scrambled siRNA-transfected cells + SEM. (C and D) MDMs from rs7554511 AA and CC ( $n=8 /$ genotype) carriers were treated with $100 \mu \mathrm{g} / \mathrm{ml}$ MDP for 15 minutes and assessed for (C) p-ERK, p-p38, p-JNK, or (D) p-IKB $\alpha$. Left: Representative flow cytometry plots. Right: Summarized data + SEM. Tx, treatment. ${ }^{*} P<0.05 ;{ }^{\circledR} P<0.001 ;{ }^{\dagger} P<1 \times 10^{-4} ;{ }^{\ddagger} P<1 \times 10^{-5}$; determined by 2-tailed Student's $t$ test.

14-3-3 $\tau$ in the recruitment of p-ERK to INAVA. Consistently, both NOD2-induced ERK activation (Figure 7E) and cytokine secretion

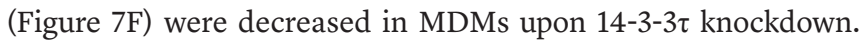
We did not observe an rs7554511 INAVA genotype-dependent difference in 14-3-3 $\tau$ recruitment to INAVA when immunoprecipitating equivalent levels of INAVA (data not shown), consistent with this being a noncoding variant that modulates expression rather than structure of INAVA. 14-3-3 $\tau$ was also recruited to INAVA with TLR4 stimulation (Supplemental Figure 18A) and 14-3-3 $\tau$ was required for optimal TLR4-induced cytokines (Supplemental Figure 18B). Therefore, upon NOD2 stimulation, INAVA assembles in a complex with proximal signaling molecules that participate in NOD2-induced outcomes.

To definitively establish the role of the 14-3-3 regions in INAVA in regulating INAVA-mediated outcomes, we generated INAVA constructs in which the serine required for 14-3-3 recruitment in each of the 3 putative 14-3-3 regions identified in INAVA (Figure 7B) was mutated to an alanine, alone and in combination. HEK293 cells did not express endogenous INAVA (Supplemental Figure 19A), such that we used these cells for our transfection studies. Each of the INAVA variants was expressed to equivalent levels in HEK293 cells (Supplemental Figure 19B). We first estab-

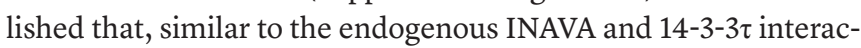
tions we observed in primary human MDMs, upon MDP treatment of HEK293 cells transfected with NOD2 and WT INAVA, 14-3-3 $\tau$ recruitment to INAVA increased and p-ERK was recruited to INAVA (Figure 7G). Importantly, mutation of the serine in each of the 14-3-3 recruitment regions in INAVA led to decreased 14-3-3 $\tau$ and p-ERK recruitment to INAVA upon NOD2 stimulation (Figure 7G). The INAVA variant in which all three 14-3-3 recruitment regions were mutated demonstrated a greater impairment in 14-3-3 $\tau$ and p-ERK recruitment (Figure $7 G$ ). Furthermore, the INAVA variants with mutations in each of the three 14-3-3 recruitment regions led to decreased NOD2-induced signaling in the pathways we found to be regulated by INAVA, with a decrease in the MAPK pathway as assessed by AP- 1 luciferase activity, and in the NF- $\kappa$ B pathway as assessed by NF- $\mathrm{KB}$ luciferase activity (Figure $7 \mathrm{H}$ ). Finally, upon MDP treatment of NOD2-transfected cells, IL-6 secretion was enhanced by WT INAVA, but this enhancement was impaired by each of the 3 INAVA mutants in the 14-3-3 recruitment regions, and further impaired in the variant with mutation of all three 14-3-3 recruitment regions (Figure 7I). Therefore, the 14-3-3 recruitment regions in INAVA are required for optimal association between INAVA and 14-3-3 $\tau$, and for PRR-induced recruitment of the signaling complex to INAVA, cellular signaling pathway activation, and cytokine secretion.

INAVA is required for optimal induction of bacterial clearance pathways and intracellular bacterial clearance. Impaired bacterial 
A

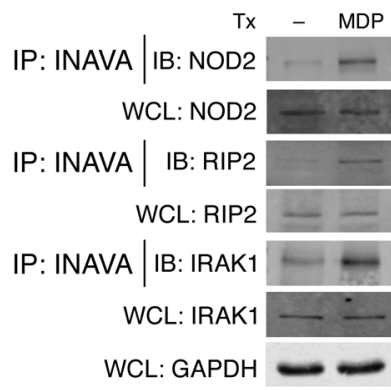

D

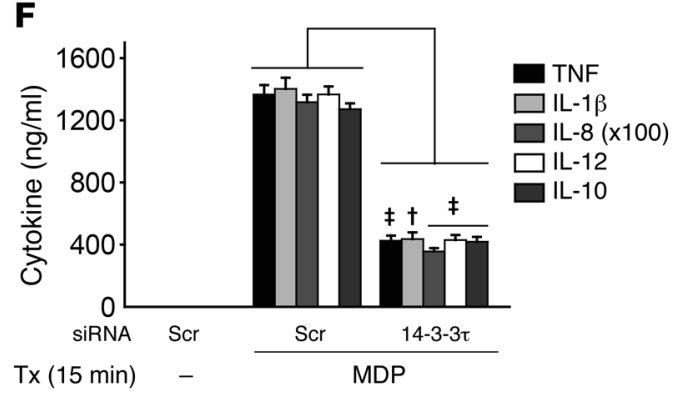

B

11 INAVA

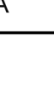

Human

Chimpanzee

Mouse

Zebrafish

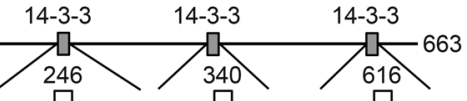

RRNSEPPP RKSSEP RVRSLP

RRNSEPPP RKS SEP

RRNSEEPPP RKSSEL

RS.SPRP. RS.SSP
RVRSLP

RVRSLP

RIRNVP
C

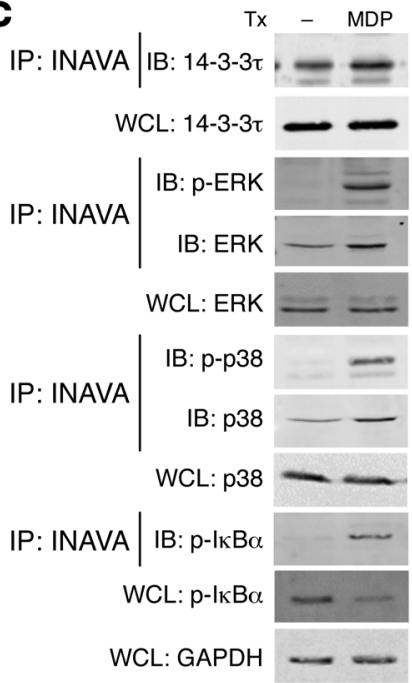

E

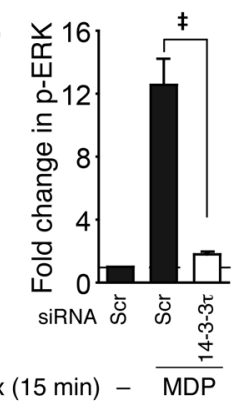

G
H

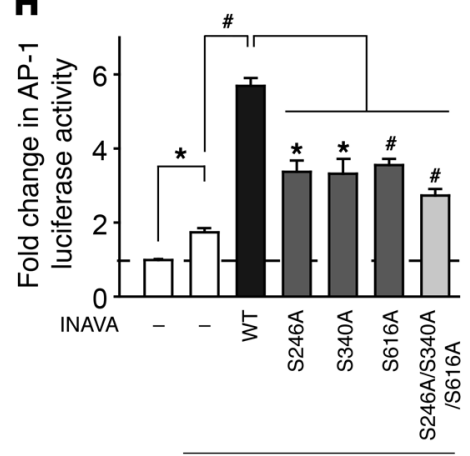

Tx (6 h)

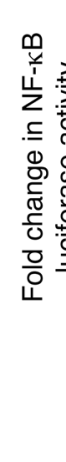

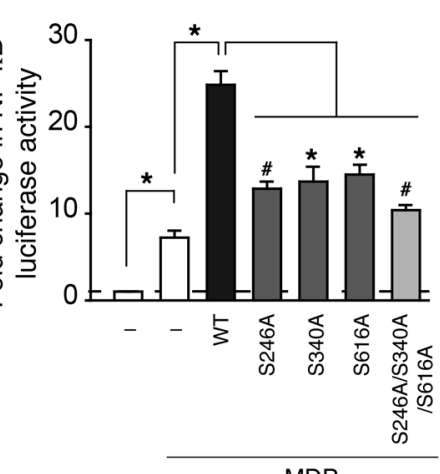

I

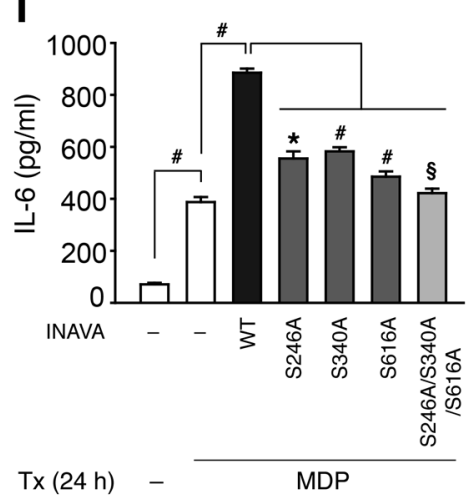

Figure 7. 14-3-3 $\tau$ recruitment to INAVA contributes to optimal assembly of a signaling complex and to INAVA modulation of PRR-induced signaling and cytokine secretion. (A) MDMs were treated with $100 \mu \mathrm{g} / \mathrm{ml}$ MDP for 15 minutes. INAVA was immunoprecipitated and recruitment of NOD2, RIP2, and IRAK1 was assessed by Western blot. Equivalent expression for the respective proteins is shown in whole-cell lysates (WCLs). (B) Sequence alignments for putative 14-3-3 binding regions within INAVA from select species. (C) MDMs were treated with $100 \mu \mathrm{g} / \mathrm{ml}$ MDP for 15 minutes. INAVA was immunoprecipitated and recruitment of $14-3-3 \tau$, p-ERK, ERK, p-p38, p38, and $p-I_{\kappa} B \alpha$ was assessed by Western blot. Equivalent expression for the respective proteins is shown in WCLs. Data are representative of $n=9$ for $14-3-3 \tau, n=9$ for $p-E R K, n=4$ for ERK, $n=3$ for $p-p 38, n=4$ for $p 38$, and $n=3$ for $p-I \kappa B \alpha$. (D-F) MDMs were transfected with scrambled or 14-3-3 $\tau$ siRNA. (D) Transfected MDMs were treated with $100 \mu \mathrm{g} / \mathrm{ml}$ MDP for 15 minutes. INAVA was immunoprecipitated and recruitment of 14-3-3 $\tau$ and $p$-ERK was assessed by Western blot. Representative Western blot for 1 of 6 , and 1 of 4 individuals, respectively. (E) Transfected cells were treated with $100 \mu \mathrm{g} / \mathrm{ml} \mathrm{MDP}$ for 15 minutes and assessed for ERK activation by phospho-flow. Fold p-ERK induction was normalized to untreated, scrambled siRNA-transfected cells $+\operatorname{SEM}(n=8)$. Similar results were observed in an additional $n=8$. (F) Transfected cells were treated with $100 \mu$ g/ml MDP for 24 hours. Mean cytokines + SEM $(n=4)$. Similar results were observed in an additional $n=8$. (G) HEK293 cells were transfected with NOD2 + the indicated HA-INAVA variants, and then treated with $100 \mu \mathrm{g} / \mathrm{ml}$ MDP for 15 minutes. HA-INAVA was immunoprecipitated with an anti-HA antibody and recruitment of 14-3-3 $\tau$ and $p-E R K$ was assessed by Western blot. Representative of $n=6(14-3-3 \tau)$ and $n=3$ (p-ERK). (H and I) HEK293 cells were transfected with NOD2, a Renilla reporter, AP-1 or NF- $\kappa B$ luciferase reporters, and empty vector or the indicated INAVA variants. (H) Transfected cells were treated with $100 \mu \mathrm{g} / \mathrm{ml}$ MDP for 6 hours and activation of AP-1 and NF- $\kappa$ B luciferase reporters was assessed and normalized to Renilla. Mean + SEM for triplicates. Representative of 3 independent experiments. (I) Transfected cells were treated with $100 \mu \mathrm{g} / \mathrm{ml}$ MDP for 24 hours and secreted IL-6 was assessed + SEM for triplicates. Representative of 3 independent experiments. Tx, treatment. ${ }^{*} P<0.05$; ${ }^{\#} P<0.01$; ${ }^{\circledR} P<0.001 ;{ }^{\dagger} P<1 \times 10^{-4}$; ${ }^{\ddagger} P<1 \times 10^{-5}$; determined by 2 -tailed Student's $t$ test. IP, immunoprecipitated; IB, immunoblotted. 
A
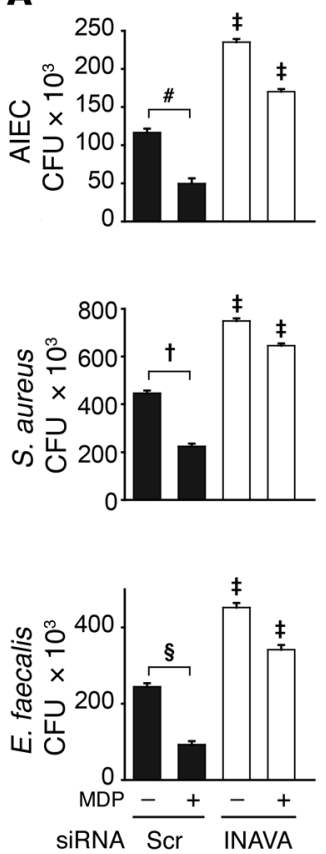

B
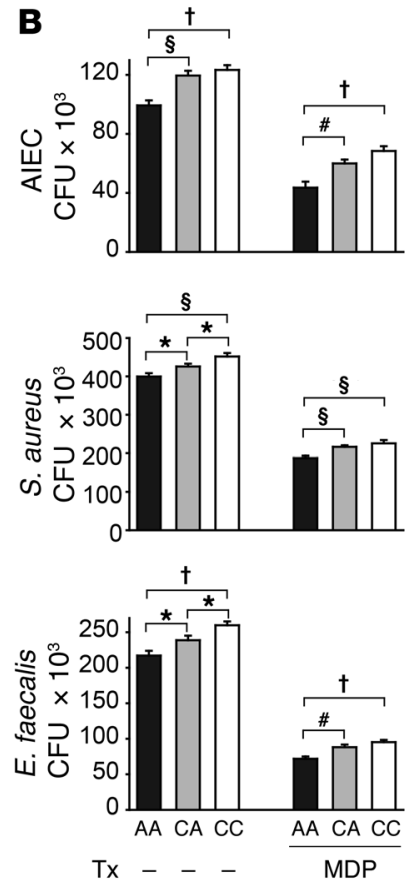

C
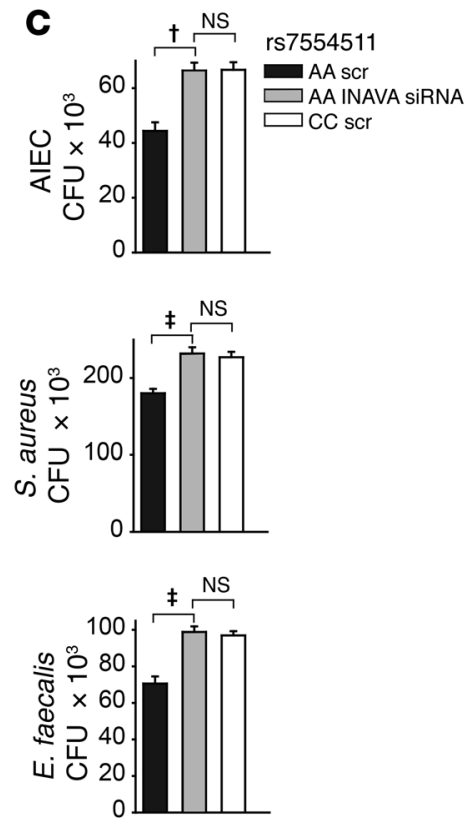

D
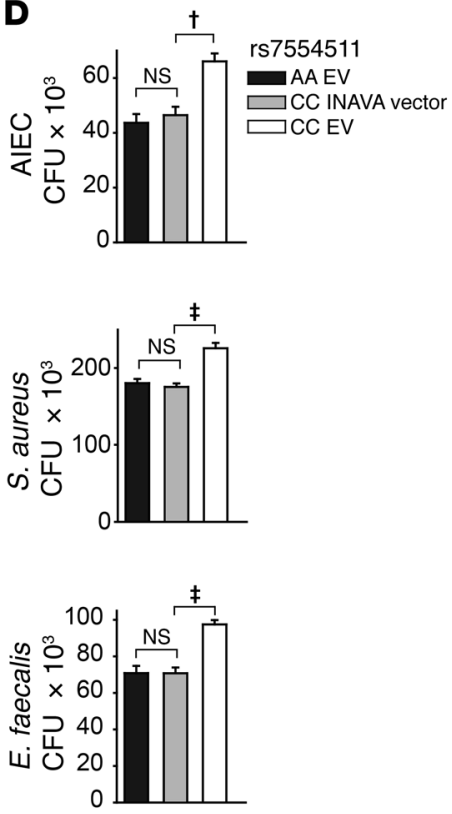

Figure 8. INAVA is required for optimal intracellular bacterial clearance and this clearance is decreased in MDMs from INAVA rs7554511 C risk carriers. (A) MDMs $(n=4)$ were transfected with scrambled or INAVA siRNA, then left untreated or treated with $100 \mu \mathrm{g} / \mathrm{ml}$ MDP for 48 hours, and then cocultured with AIEC, S. aureus, or E. faecalis. Shown are bacterial colony forming units (CFU) + SEM. Significance is compared with scrambled siRNA-transfected cells for the corresponding treatment or as indicated. Similar results were observed in an additional $n=4$. (B) MDMs from rs7554511 AA, CA, CC carriers ( $n$ $=15$ /genotype) were left untreated or treated with $100 \mu \mathrm{g} / \mathrm{ml}$ MDP for 48 hours and then cocultured with AIEC, S. aureus, or E. faecalis. Shown are the CFU + SEM. (C and D) MDMs from rs7554511 CC or AA carriers ( $n=15 /$ genotype) were transfected with (C) scrambled or INAVA siRNA, or (D) empty vector (EV) or INAVA vector, treated with $100 \mu \mathrm{g} / \mathrm{ml}$ MDP for 48 hours, and then cocultured with the indicated bacteria. Shown are CFU + SEM. NS, not significant; scr, scrambled; Tx, treatment. ${ }^{*} P<0.05$; ${ }^{\#} P<0.01$; ${ }^{\circledR} P<0.001$; ${ }^{\dagger} P<1 \times 10^{-4}$; ${ }^{\ddagger} P<1 \times 10^{-5}$; determined by 2-tailed Student's $t$ test.

clearance can increase the risk for IBD $(5,36-38)$, and PRR-initiated pathways contribute to intracellular bacterial clearance (2). As the rs7554511 IBD risk variant leads to reduced INAVA expression and reduced PRR-induced signaling and cytokines, we questioned if INAVA was required for optimal macrophage-mediated bacterial clearance. Prolonged stimulation of macrophages through NOD2 can enhance the ability of macrophages to clear bacteria and simulates the ongoing PRR stimulation conditions that intestinal macrophages encounter (39). We therefore examined the role of INAVA in bacterial clearance in both untreated and chronic NOD2-stimulated human MDMs. INAVA was required for optimal clearance of adherent invasive Escherichia coli (AIEC), bacteria colonizing the ilea of Crohn's disease patients with increased frequency (40), in both untreated and chronic NOD2-stimulated macrophages (Figure 8A). Similar results were observed with 2 resident bacteria, Staphylococcus aureus and Enterococcus faecalis (Figure 8A). We then questioned how the INAVA rs7554511 risk genotype regulates bacterial clearance. Consistent with decreased PRR-initiated outcomes (Figure 1, Supplemental Figure 1, and Figure 6, C and D), MDMs from rs7554511 C risk carriers showed decreased baseline and NOD2-induced clearance of each of the 3 bacteria compared with AA carrier MDMs (Figure 8B). Upon knockdown of INAVA expression in rs7554511 AA carriers to the levels observed in CC carriers (Supplemental Figure 8A), NOD2induced bacterial clearance was decreased to the levels of CC carriers (Figure 8C). In contrast, increasing INAVA expression in rs7554511 CC carriers to the levels observed in AA carriers (Supplemental Figure 9) increased bacterial clearance to the levels observed in AA carriers (Figure 8D), thereby highlighting that INAVA expression modulation accounted for the rs7554511 genotype-dependent regulation of bacterial clearance. Taken together, these results show that MDMs from rs7554511 CC risk carriers exhibit decreased bacterial clearance.

We next considered how INAVA might be modulating mechanisms contributing to bacterial clearance, including reactive oxygen species (ROS), reactive nitrogen species (RNS), and autophagy pathways. Upon NOD2 stimulation of MDMs, INAVA knockdown resulted in decreased ROS production (Figure 9A). To define mechanisms for this reduced ROS production we examined members of the NADPH complex required for cellular ROS and found decreased induction of p40phox, p47phox, and p67phox mRNA with INAVA knockdown (Figure 9B). Induction of the RNS-inducing enzyme NOS 2 was also decreased (Figure 9C). Each of these proteins was required for optimal AIEC clearance (Supplemental Figure 20, A and B). We also observed a requirement for INAVA in the NOD2-induced autophagy observed in MDMs (Figure 9D). This was associated with an INAVA-dependent role for induction of the autophagy-associated gene, ATG5, whereas induction of ATG10 and immunity-related GTPase M (IRGM) expression was not INAVA dependent (Figure 9E). We verified that ATG5 was required for optimal NOD2-induced autophagy (Supplemental Figure 20, C and D) and bacterial clearance (Supplemental Figure 
A

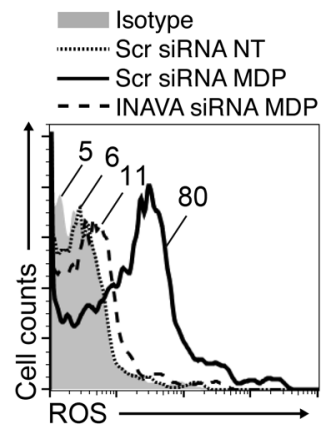

B
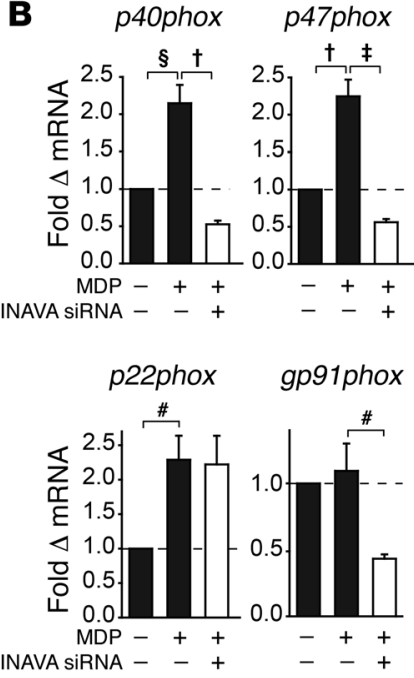
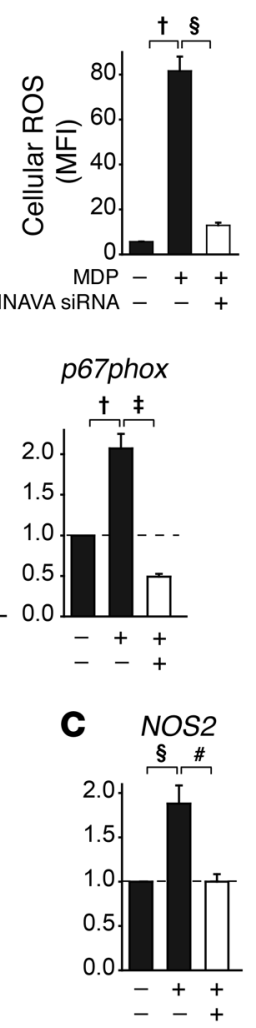

D

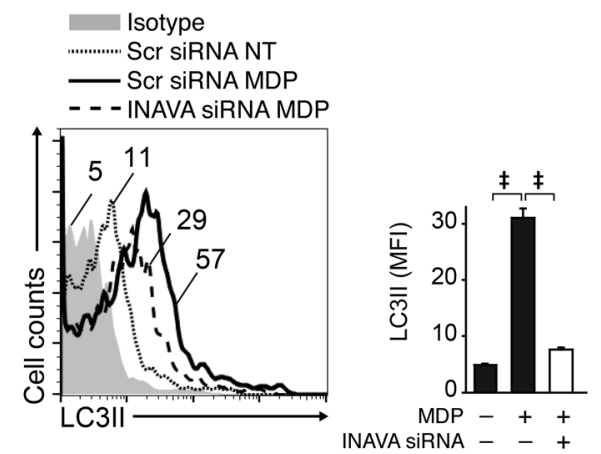

$\mathbf{E}$
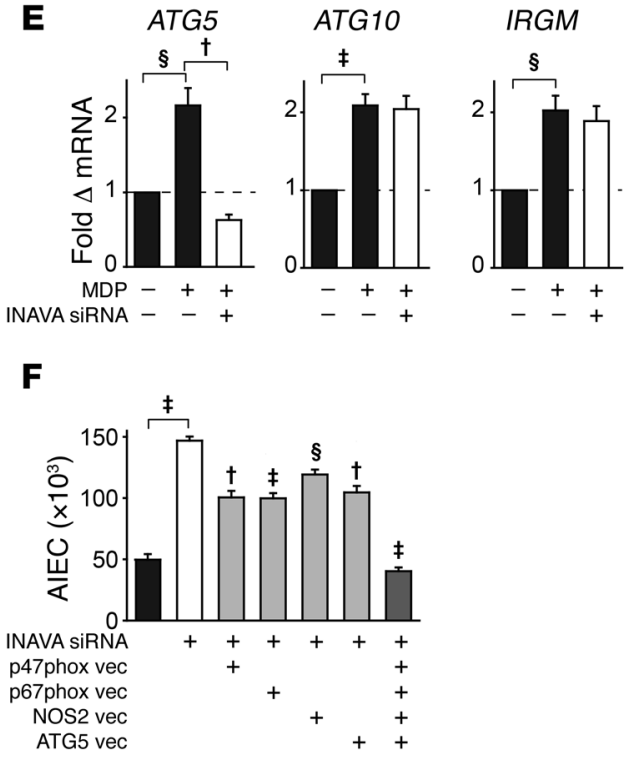

Figure 9. PRR-induced ROS, RNS, and autophagy are required for INAVA-mediated bacterial clearance. (A-E) MDMs were transfected with scrambled or INAVA siRNA and then left untreated or treated with $100 \mu \mathrm{g} / \mathrm{ml} \mathrm{MDP}$ for 48 hours. (A and D) Cells were analyzed by flow cytometry utilizing (A) the ROSdetecting dye $\mathrm{H}_{2}$ DCDFDA $(n=4)$ or (D) LC3II antibody $(n=4)$. Shown are representative flow cytometry plots with mean fluorescence intensity (MFI) values as indicated and a summary graph with MFI + SEM. (B, C, and E) mRNA expression was assessed by quantitative reverse transcription PCR. Data are represented as the fold mRNA induction compared with scrambled siRNA-transfected, untreated cells $(n=8$, similar results seen in an additional $n=4)+$ SEM. (F) MDMs $(n=6)$ were transfected with scrambled or INAVA siRNA \pm p47phox-, p67phox-, NOS2-, or ATC5-expressing vectors alone or in combination or with empty vector, then left untreated or treated with $100 \mu \mathrm{g} / \mathrm{ml} \mathrm{MDP}$ for 48 hours. Cells were cocultured with AIEC at 10:1 MOI. Shown are bacterial CFU + SEM. Scr, scrambled; vec, vector. ${ }^{\sharp} P<0.01 ;{ }^{\circledR} P<0.001 ;{ }^{\dagger} P<1 \times 10^{-4} ;{ }^{\ddagger} P<1 \times 10^{-5}$; determined by 2-tailed Student's $t$ test.

20E). To clearly address the role of these pathways downstream of INAVA upon NOD2 stimulation, we restored p47phox and p67phox, the NADPH oxidase members showing the strongest contribution to AIEC clearance (Supplemental Figure 20B), in INAVA-deficient MDMs (Supplemental Figure 20F). This rescued ROS in INAVA-deficient cells (Supplemental Figure 20G) and partially rescued intracellular bacterial clearance (Figure 9F). Restoring NOS2 in INAVA-deficient MDMs (Supplemental Figure 20H) similarly partially rescued bacterial clearance (Figure 9F). Restoring ATG5 expression in INAVA-deficient macrophages (Supplemental Figure 20I) rescued autophagy (Supplemental Figure 20J) and partially rescued bacterial clearance (Figure 9F). Restoring ROS, RNS, and autophagy pathways in combination in INAVAdeficient MDMs fully rescued bacterial clearance, highlighting cooperation between these pathways (Figure 9F).

Consistent with the INAVA genotype-dependent regulation of bacterial killing, NOD2-mediated induction of ROS and the NAPDH oxidase subunits p47phox and p67phox (Figure 10A),
NOS2 (Figure 10B), as well as the induction of LC3II and ATG5 (Figure 10C) was increased in MDMs from rs7554511 AA carriers compared with CC carriers. Heterozygotes demonstrated an intermediate phenotype. Importantly, knocking down INAVA expression in AA carriers to the levels seen in CC carriers (Supplemental Figure 8A) reduced each of these outcomes to the levels of CC carriers (Figure 10, D-F). Conversely, increasing the INAVA expression in CC carriers to the levels seen in AA carriers (Supplemental Figure 9A) increased each of the bacterial clearance pathways to the levels seen in AA carriers (Figure 10, G-I).

INAVA regulates a broad range of NOD2-induced transcripts. As INAVA regulates both NOD2-induced MAPK and NF- $\mathrm{KB}$ pathways, and these signaling pathways in turn regulate a broad range of NOD2-induced transcripts, we postulated that in addition to its regulation of PRR-induced cytokines and the select antimicrobial-associated pathways we had examined, INAVA would regulate a broad spectrum of NOD2-induced transcripts. We focused on the NOD2-upregulated transcripts identified 

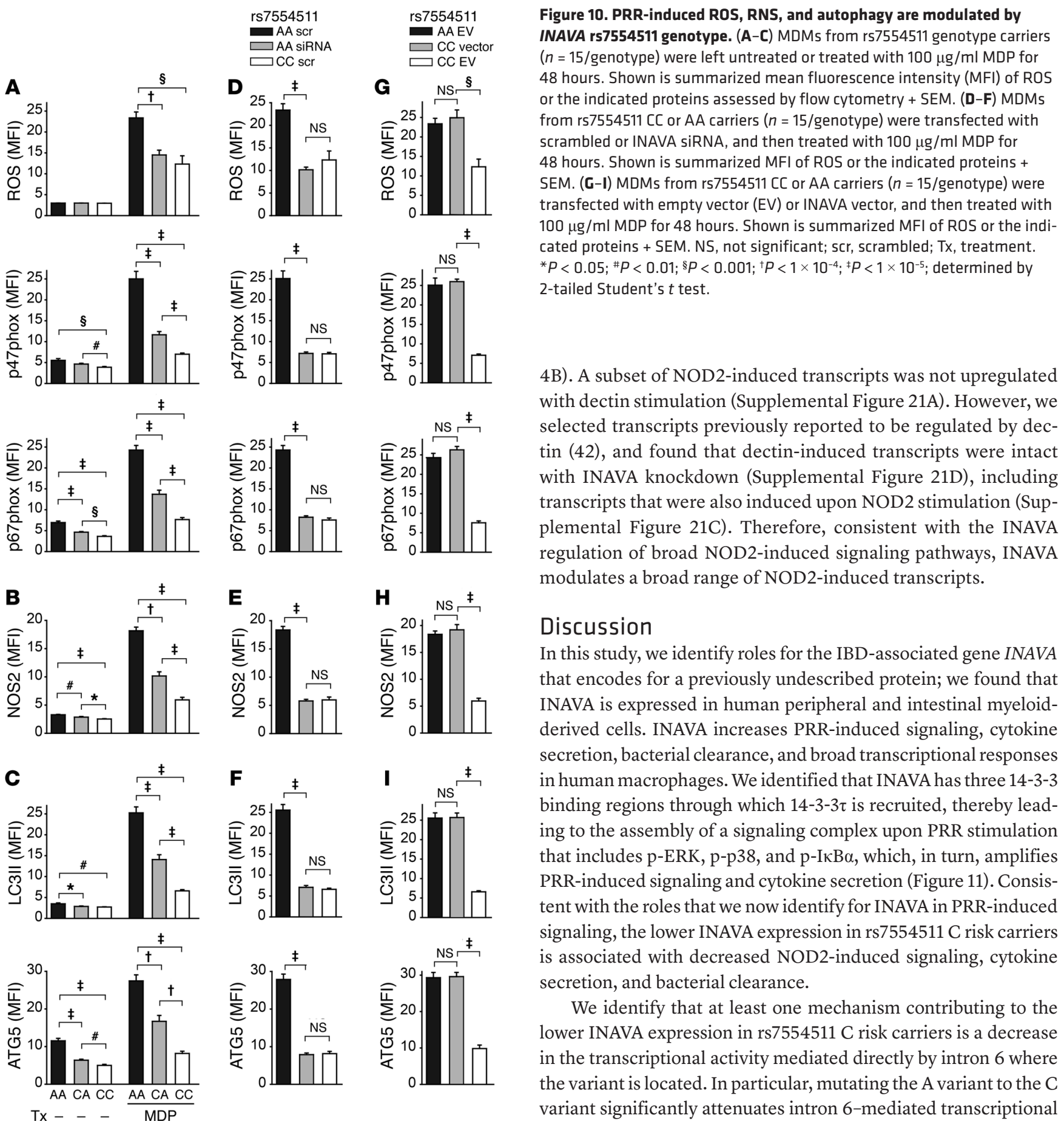

through microarray (41), and found that the upregulation of these transcripts was impaired with INAVA knockdown (Supplemental Figure 21A). To demonstrate the MAPK/NF- $\mathrm{KB}$ dependency of these transcripts, we examined these same transcripts upon NOD2 stimulation while inhibiting the MAPK and NF-kB pathways and found that their upregulation was similarly impaired (Supplemental Figure 21A). We ensured the cells were viable under these conditions (Supplemental Figure 21B). As a control for specificity of INAVA effects, we examined dectin-induced transcripts, as we had found that dectin-induced antiinflammatory mediators did not depend on INAVA (Supplemental Figure
Figure 10. PRR-induced ROS, RNS, and autophagy are modulated by INAVA rs7554511 genotype. (A-C) MDMs from rs7554511 genotype carriers 48 hours. Shown is summarized mean fluorescence intensity (MFI) of ROS or the indicated proteins assessed by flow cytometry + SEM. (D-F) MDMs 48 hours. Shown is summarized MFI of ROS or the indicated proteins SEM. (G-I) MDMs from rs 7554511 CC or AA carriers ( $n=15$ /genotype) were transfected with empty vector (EV) or INAVA vector, and then treated with cated proteins + SEM. NS, not significant; scr, scrambled; Tx, treatment. 2-tailed Student's $t$ test.

4B). A subset of NOD2-induced transcripts was not upregulated with dectin stimulation (Supplemental Figure 21A). However, we selected transcripts previously reported to be regulated by dectin (42), and found that dectin-induced transcripts were intact with INAVA knockdown (Supplemental Figure 21D), including transcripts that were also induced upon NOD2 stimulation (Supplemental Figure 21C). Therefore, consistent with the INAVA regulation of broad NOD2-induced signaling pathways, INAVA modulates a broad range of NOD2-induced transcripts.

\section{Discussion}

In this study, we identify roles for the IBD-associated gene INAVA that encodes for a previously undescribed protein; we found that INAVA is expressed in human peripheral and intestinal myeloidderived cells. INAVA increases PRR-induced signaling, cytokine secretion, bacterial clearance, and broad transcriptional responses in human macrophages. We identified that INAVA has three 14-3-3 binding regions through which $14-3-3 \tau$ is recruited, thereby leading to the assembly of a signaling complex upon PRR stimulation that includes p-ERK, p-p38, and p-IкB $\alpha$, which, in turn, amplifies PRR-induced signaling and cytokine secretion (Figure 11). Consistent with the roles that we now identify for INAVA in PRR-induced signaling, the lower INAVA expression in rs $7554511 \mathrm{C}$ risk carriers is associated with decreased NOD2-induced signaling, cytokine secretion, and bacterial clearance.

We identify that at least one mechanism contributing to the lower INAVA expression in rs7554511 C risk carriers is a decrease in the transcriptional activity mediated directly by intron 6 where the variant is located. In particular, mutating the $\mathrm{A}$ variant to the $\mathrm{C}$ variant significantly attenuates intron 6-mediated transcriptional activity. This is likely attributable to a decreased cooperative effect between multiple transcription factors. We identified that at least one of the transcription factors contributing to intron 6-mediated transcriptional activity is TBP, and that TBP-induced transcription is less in the rs7554511 C variant. It is also possible that there are additional polymorphisms in the INAVA region in LD with rs7554511 that affect regulatory regions that also modulate INAVA expression, such that the rs7554511 genotype-dependent regulation of INAVA is likely multifactorial.

Decreased cytokines and PRR-initiated outcomes (9-11, 17, $18,43,44)$ and impaired bacterial clearance have been implicated in subsets of IBD patients $(5,36-38,45-47)$. Furthermore, genetic perturbations in pathways critical for bacterial clearance have 


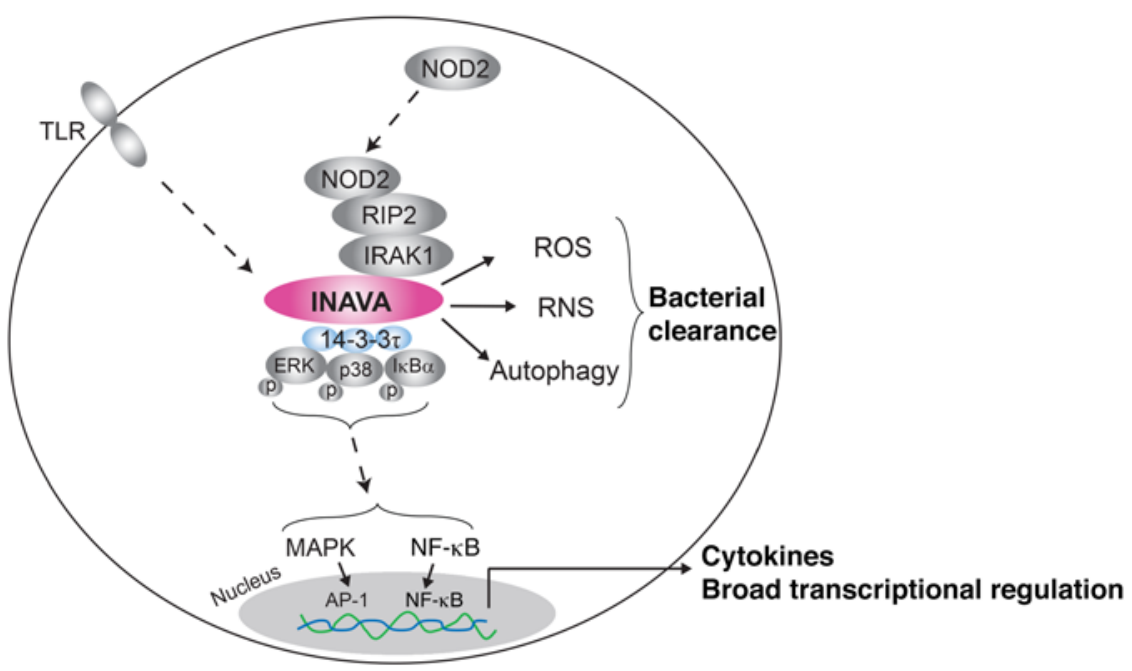

Figure 11. Model of INAVA mechanisms for regulating PRR-induced signaling, cytokines, and bacterial clearance. NOD2 stimulation in MDMs results in the assembly of a complex that includes NOD2, RIP2, IRAK1, INAVA, and 14-3-3 $\tau$. INAVA contains 3 distinct 14-3-3 binding domains, and $14-3-3 \tau$ recruitment, in turn, modulates the recruitment of additional signaling molecules to INAVA, including $p$-ERK, $p-p 38$, and $p-1 \kappa B \alpha$, which then contribute to activation of downstream signaling pathways and cytokine secretion. In addition to the induction of cytokines, INAVA is required for induction of a broad range of NOD2-dependent transcripts, as well as for optimal bacterial clearance pathways and intracellular bacterial clearance in MDMs. Importantly, INAVA is required for optimal signaling and cytokine secretion downstream of multiple PRRs. MDMs from IBD rs 7554511 C risk carriers in INAVA demonstrate lower INAVA expression, and consistently, decreased MAPK and NF- $\kappa B$ signaling, cytokine secretion, and bacterial clearance.

been well described to be associated with early-onset $\operatorname{IBD}(36,37$, 48). The decreased responses through PRR-initiated pathways in INAVA risk carriers may also contribute to impaired recruitment of immune cells and other critical innate functions, including innateinstructed adaptive immune outcomes. Moreover, INAVA may contribute to functions in myeloid-derived cells in addition to those initiated by PRR stimulation, as well as to functions by additional cell subsets. We did not observe that expression of other genes in the region was modulated in an rs7554511 genotype-dependent manner in myeloid-derived cells or that these genes were able to modulate NOD2-induced cytokines. However, it remains possible that a subset of these other genes, or genes beyond the $500-\mathrm{kb}$ distance from the rs7554511 polymorphism that we examined, may regulate other PRR-dependent or PRR-independent functions in myeloid-derived cells or alternative functions in nonmyeloid cell subsets in a genotype-dependent manner. Moreover, we focus on a common risk variant in INAVA; there are rare INAVA variants (49) that may result in consequences to INAVA function through alternative mechanisms.

We identified that INAVA assembled in a complex with several proteins upon PRR stimulation, including NOD2, RIP2, IRAK1, and $14-3-3 \tau$. A prior study found that $14-3-3 \sigma$ interacts with INAVA (C1orf106) in a yeast 2-hybrid system (50). Only a few studies have identified a role for 14-3-3 proteins in modulating signaling downstream of PRRs (31-33). 14-3-3 isoforms have been shown to associate with molecules that affect PRR signaling pathways $(50,51)$. One such association occurs between $14-3-3$ isoforms $\varepsilon, \zeta$, and $\eta$ and TPL2 (33), another IBD-associated gene (12); these 14-3-3/TPL2 interactions are necessary for PRR-induced ERK activation. While current studies have not implicated 14-3-3 genetic associations with IBD, the DisGENET database shows that various 14-3-3 isoforms are associated with squamous cell carcinoma, multiple sclerosis, and schizophrenia. Interestingly, a recent study using genome-wide interaction analysis identified an interaction between the INAVA (C1orf106) locus on chromosome 1 and the $T E C$ locus on chromosome 4 with respect to Crohn's disease risk (52). TEC family kinase proteins have recently been found to regulate TLR-mediated signaling in myeloid cells (53), which is intriguing given the important role we identify for INAVA in PRR-mediated outcomes. In future studies, it would be interesting to examine if TEC and INAVA cooperate in a functional manner to mediate PRRinduced outcomes.

While we observe that MDMs from rs7554511 heterozygotes show functional outcomes closer to those of $\mathrm{CC}$ carriers for some measures (such as NOD2-induced cytokines in Figure 1), they show an intermediate phenotype for other readouts (e.g., bacterial clearance mechanisms; Figures 9 and 10). One reason for this might be a different INAVA expression threshold required for distinct immunological outcomes. Expression/function threshold differences have been observed for various molecules $(54,55)$.

Our findings define a critical role for the newly defined protein INAVA in processes crucial for intestinal immune homeostasis, including the regulation outcomes downstream of a broad range of PRRs in human macrophages. We further identify that in MDMs from INAVA rs7554511 C IBD risk individuals PRR-induced signaling, cytokines, and bacterial clearance are decreased, thereby highlighting that modulation of INAVA levels may provide a therapeutic benefit in intestinal inflammation and human IBD.

\section{Methods}

Patient recruitment and genotyping. Informed consent was obtained per protocol approved by the IRB at Yale University and healthy controls were recruited for the studies. We performed genotyping by TaqMan genotyping (Applied Biosystems) or utilizing the Sequenom platform (Sequenom Inc.).

MDM cell isolation and cell culture. Monocytes were purified from human peripheral blood mononuclear cells by positive CD14 selection (Miltenyi Biotec) or adhesion, tested for purity, and cultured with $10 \mathrm{ng} / \mathrm{ml}$ M-CSF (Shenandoah Biotechnology) for MDM differentiation (20). Cultured myeloid cells were treated with MDP (Bachem), $\mathrm{Pam}_{3} \mathrm{Cys}$ (EMD Millipore), lipid A (Peptides International), polyI:C, flagellin, CL097, or CpG DNA (all Invivogen). Supernatants were assayed for TNF, IL-6, IL-8, IL-10 (all BD Biosciences), IL-12, or IL-1 $\beta$ (both eBioscience) by ELISA. 
Intestinal lamina propria cell isolation. Intestinal lamina propria cells were isolated from colonic resection specimens from uninvolved intestine in 7 non-IBD patients undergoing surgery for diverticular disease or colon cancer (9).

$m R N A$ expression analysis. RNA was isolated, reverse transcribed, and quantitative PCR performed on the ABI Prism 7000 (Applied Biosystems) using primer sequences as per Supplemental Table 1. Samples were normalized to GAPDH.

Transfection of siRNAs and DNA vectors. Pooled siRNA containing 4 different siRNAs at $100 \mathrm{nM}$ or indicated concentrations for each INAVA (C1orf106), 14-3-3 $\tau$, TBP, or scrambled siRNA (SMARTpool, Dharmacon), or of vectors expressing ATG5 (Addgene plasmid 24922; deposited by Toren Finkel; see ref. 56), NOS2 (gift of Tony Eissa; see ref. 57), p47phox and p67phox (gifts of Celine DerMardirossian; see ref. 58), or pEGFP-C1-TBP (Addgene plasmid 26674; deposited by Sui Huang; see ref. 59) were transfected into MDMs using a Nucleofector Kit (Amaxa) for 48 hours. INAVA (C1orf106) was subcloned from BC106877 (ORD3016) plasmid (Transomic) into pcDNA3.0 along with an HA tag. HA-INAVA S246A, HA-S340A, and HA-S616A, alone and in combination, were generated through site-directed mutagenesis (QuikChange Lightning Kit; Agilent Technologies). INAVA intron 6 containing the rs7554511 A SNP was subcloned into the pGL4.17 vector (Promega). Site-directed mutagenesis was used to generate the rs $7554511 \mathrm{C}$ variant. For INAVA transfection assays, $800 \mathrm{ng}$ of each HA-INAVA construct was transfected along with $50 \mathrm{ng}$ NOD2 $\pm 50 \mathrm{ng}$ pNF-кB-luciferase or pAP-1-luciferase (both Clontech), and 15 ng pRLCMV (Promega) as a Renilla normalization control. For transcription factor transfections, 50 ng of each INAVA intron 6 construct, 800 ng pEGFP-C1-TBP, and 15 ng pRLCMV as a Renilla normalization control was transfected into cells. Numbering of the nucleotides and amino acids was according to the Genbank accession number BC106877.2.

Protein expression analysis. INAVA was immunoprecipitated from MDMs with antibodies against INAVA (C1orf106) (Abcam, ab121945) or from transfected HEK293 cells with antibodies against HA (Abcam, ab137838) bound to protein A or protein G Sepharose (EMD Millipore). Immunoprecipitates were blotted for NOD2 (Cayman Chemicals, 160777), RIP2 (BD Biosciences, clone 25/RIG-G), 14-3-3 (Abcam, ab10439), p-ERK (catatog 9101, clone E10), ERK (clone 137F5), p-p38 (clone 28B10), $\mathrm{p}-\mathrm{I} \kappa \mathrm{B} \alpha$ (clone 14D4), I $\mathrm{KB} \alpha$ (catalog 9242), or IRAK1 (clone D51G7) (all Cell Signaling Technology) and p38 (Santa Cruz Biotechnology, clone A-12) (20). GAPDH (EMD Millipore) or the respective protein in whole-cell lysate served as the loading control.
Phosphoprotein induction was determined using Alexa Fluor 647-, phycoerythrin-, Alexa Fluor 488-, or biotin-labeled antibodies against p-ERK (clone D13.14.4E), p-p38 (clone 3D7), p-JNK (clone G9), or $\mathrm{p}-\mathrm{I} \kappa \mathrm{B} \alpha$ (clone 14D4) (all Cell Signaling Technology) by flow cytometry. Permeabilized cells were assessed for protein expression by flow cytometry with anti-INAVA (anti-C1orf106), anti-ATG5 [clone EPR1755(2)] (both Abcam), anti-NOS2 (catalog 2977), anti-LC3II (catalog 2775) (both Cell Signaling Technology), anti-p47phox (clone A-7), and antip67phox (clone D-6) (both Santa Cruz Biotechnology). Isotype controls matching the specific antibody were used in the flow cytometry.

Intracellular ROS measurement. ROS was measured by flow cytometry using $10 \mu \mathrm{M}$ 2',7'-dichlorodihydrofluorescein diacetate ( $\mathrm{H}_{2}$ DCFDA) (Invitrogen).

Intracellular bacterial clearance. Following treatment, MDMs were infected in triplicate for 1 hour with AIEC strain LF82 (a gift from Emiko Mizoguchi, Kurume University, Kurume, Japan) at 10:1 MOI, S. aureus at 1:1 MOI or E. faecalis at 10:1 MOI, washed 3 times with PBS, and incubated in HBSS medium containing $50 \mu \mathrm{g} / \mathrm{ml}$ gentamicin for an additional hour. Cells were washed, lysed with $1 \%$ Triton X-100 (Sigma-Aldrich), and plated on MacConkey or LB agar.

Luciferase activity. Cells were lysed, assayed for luciferase, and normalized to Renilla activity (Promega) according to the manufacturer's instructions and using the Synergy 2 (BioTek).

Statistics. Significance was assessed using a 2-tailed Student's $t$ test. To keep cytokines on the same axis, a multiplier was applied for the higher levels of IL-8 as shown in the Figure keys. $P$ less than 0.05 was considered significant.

\section{Author contributions}

JY, MH, and CA were involved in research design, conducting experiments, analyzing data, and writing the manuscript.

\section{Acknowledgments}

This work was supported by the NIH grants DK099097, DK062422, and DK-P30-34989, The Broad Foundation, and The Crohn's and Colitis Foundation of America. We thank Tony Eissa, Celine DerMardirossian, and Emiko Mizoguchi for reagents.

Address correspondence to: Clara Abraham, Department of Internal Medicine, Section of Digestive Diseases, 333 Cedar Street (LMP 1080), New Haven, Connecticut 06520, USA. Phone: 203.785.5610; E-mail: clara.abraham@yale.edu.
1. Abraham C, Cho JH. Inflammatory bowel disease. NEngl JMed. 2009;361(21):2066-2078.

2. Abraham C, Medzhitov R. Interactions between the host innate immune system and microbes in inflammatory bowel disease. Gastroenterology. 2011;140(6):1729-1737.

3. Hugot JP, et al. Association of NOD2 leucine-rich repeat variants with susceptibility to Crohn's disease. Nature. 2001;411(6837):599-603.

4. Ogura Y, et al. A frameshift mutation in NOD2 associated with susceptibility to Crohn's disease. Nature. 2001;411(6837):603-606.

5. Lassen KG, et al. Atg16L1 T300A variant decreases selective autophagy resulting in altered cytokine signaling and decreased antibacterial defense. Proc Natl Acad Sci US A.
2014;111(21):7741-7746

6. Hedl M, Abraham C. IRF5 risk polymorphisms contribute to interindividual variance in pattern recognition receptor-mediated cytokine secretion in human monocyte-derived cells. J Immunol. 2012;188(11):5348-5356.

7. Hedl M, Abraham C. A TNFSF15 disease-risk polymorphism increases pattern-recognition receptor-induced signaling through caspase8-induced IL-1. Proc Natl Acad Sci U S A. 2014;111(37):13451-13456.

8. Hedl M, Abraham C. A TPL2 (MAP3K8) diseaserisk polymorphism increases TPL2 expression thereby leading to increased pattern recognition receptor-initiated caspase- 1 caspase- 8 activation, signalling cytokine secretion. Gut.
2016;65(11):1799-1811.

9. Hedl M, Lahiri A, Ning K, Cho JH, Abraham C. Pattern recognition receptor signaling in human dendritic cells is enhanced by ICOS ligand and modulated by the Crohn's disease ICOSLG risk allele. Immunity. 2014;40(5):734-746.

10. Hedl M, Zheng S, Abraham C. The IL18RAP region disease polymorphism decreases IL18RAP/IL-18R1/IL-1R1 expression and signaling through innate receptor-initiated pathways. J Immunol. 2014;192(12):5924-5932.

11. Lamas B, et al. CARD9 impacts colitis by altering gut microbiota metabolism of tryptophan into aryl hydrocarbon receptor ligands. Nat Med. 2016;22(6):598-605.

12. Jostins L, et al. Host-microbe interactions have 
shaped the genetic architecture of inflammatory bowel disease. Nature. 2012;491(7422):119-124.

13. Franke A, et al. Genome-wide meta-analysis increases to 71 the number of confirmed Crohn's disease susceptibility loci. Nat Genet. 2010;42(12):1118-1125.

14. Liu JZ, et al. Association analyses identify 38 susceptibility loci for inflammatory bowel disease and highlight shared genetic risk across populations. Nat Genet. 2015;47(9):979-986.

15. International Multiple Sclerosis Genetics Consortium (IMSGC). Comprehensive follow-up of the first genome-wide association study of multiple sclerosis identifies KIF21B and TMEM39A as susceptibility loci. Hum Mol Genet. 2010;19(5):953-962.

16. Seok J, et al. Genomic responses in mouse models poorly mimic human inflammatory diseases. Proc Natl Acad Sci U S A. 2013;110(9):3507-3512.

17. Girardin SE, et al. Nod2 is a general sensor of peptidoglycan through muramyl dipeptide (MDP) detection. J Biol Chem. 2003;278(11):8869-8872.

18. Inohara $\mathrm{N}$, et al. Host recognition of bacterial muramyl dipeptide mediated through NOD2. Implications for Crohn's disease. J Biol Chem. 2003;278(8):5509-5512.

19. Hedl M, Abraham C. Distinct roles for Nod2 protein and autocrine interleukin-1beta in muramyl dipeptide-induced mitogen-activated protein kinase activation and cytokine secretion in human macrophages. J Biol Chem. 2011;286(30):26440-26449.

20. Hedl M, Li J, Cho JH, Abraham C. Chronic stimulation of Nod2 mediates tolerance to bacterial products. Proc Natl Acad Sci U S A. 2007;104(49):19440-19445.

21. Brown GD. Dectin-1: a signalling non-TLR pattern-recognition receptor. Nat Rev Immunol. 2006;6(1):33-43.

22. Feng BS, He SH, Zheng PY, Wu L, Yang PC. Mast cells play a crucial role in Staphylococcus aureus peptidoglycan-induced diarrhea. Am J Pathol. 2007;171(2):537-547.

23. Zheng S, Abraham C. NF- $\kappa B 1$ inhibits NOD2-induced cytokine secretion through ATF3-dependent mechanisms. Mol Cell Biol. 2013;33(24):4857-4871.

24. Park JH, et al. RICK/RIP2 mediates innate immune responses induced through Nod1 and Nod2 but not TLRs. JImmunol. 2007;178(4):2380-2386.

25. Yang Y, Yin C, Pandey A, Abbott D, Sassetti C, Kelliher MA. NOD2 pathway activation by MDP or Mycobacterium tuberculosis infection involves the stable polyubiquitination of Rip2. J Biol Chem. 2007;282(50):36223-36229.

26. Hedl M, Abraham C. Nod2-induced autocrine interleukin-1 alters signaling by ERK and $\mathrm{p} 38$ to differentially regulate secretion of inflammatory cytokines. Gastroenterology. 2012;143(6):1530-1543.

27. Opitz B, et al. Nucleotide-binding oligomerization domain proteins are innate immune receptors for internalized Streptococcus pneumoniae. J Biol Chem. 2004;279(35):36426-36432.

28. Uhart M, Bustos DM. Protein intrinsic disorder and network connectivity. The case of 14-3-3 proteins. Front Genet. 2014;5:10.

29. Reinhardt HC, Yaffe MB. Phospho-Ser/Thrbinding domains: navigating the cell cycle and DNA damage response. Nat Rev Mol Cell Biol. 2013;14(9):563-580.

30. Zhao J, Meyerkord CL, Du Y, Khuri FR, Fu H. 14-3-3 proteins as potential therapeutic targets. Semin Cell Dev Biol. 2011;22(7):705-712.

31. Faisal A, Saurin A, Gregory B, Foxwell B, Parker PJ. The scaffold MyD88 acts to couple protein kinase Cepsilon to Toll-like receptors. J Biol Chem. 2008;283(27):18591-18600.

32. Yang CS, et al. The autophagy regulator Rubicon is a feedback inhibitor of CARD9-mediated host innate immunity. Cell Host Microbe. 2012;11(3):277-289.

33. Ben-Addi A, et al. IKB kinase-induced interaction of TPL-2 kinase with 14-3-3 is essential for Toll-like receptor activation of ERK-1 and -2 MAP kinases. Proc Natl Acad Sci U S A. 2014;111(23):E2394-E2403.

34. Schuster TB, Costina V, Findeisen P, Neumaier $\mathrm{M}$, Ahmad-Nejad P. Identification and functional characterization of 14-3-3 in TLR2 signaling. JProteome Res. 2011;10(10):4661-4670.

35. Aguilera C, Fernández-Majada V, Inglés-Esteve J, Rodilla V, Bigas A, Espinosa L. Efficient nuclear export of p65-IkappaBalpha complexes requires 14-3-3 proteins. J Cell Sci. 2006; 119(Pt 17):3695-3704.

36. Dhillon SS, et al. Variants in nicotinamide adenine dinucleotide phosphate oxidase complex components determine susceptibility to very early onset inflammatory bowel disease. Gastroenterology. 2014;147(3):680-689.e2.

37. Muise AM, et al. NADPH oxidase complex and IBD candidate gene studies: identification of a rare variant in NCF2 that results in reduced binding to RAC2. Gut. 2012;61(7):1028-1035.

38. Holland SM. Chronic granulomatous disease. Clin Rev Allergy Immunol. 2010;38(1):3-10.

39. Lahiri A, Abraham C. Activation of pattern recognition receptors up-regulates metallothioneins, thereby increasing intracellular accumulation of zinc, autophagy, and bacterial clearance by macrophages. Gastroenterology. 2014;147(4):835-846.

40. Darfeuille-Michaud A, et al. High prevalence of adherent-invasive Escherichia coli associated with ileal mucosa in Crohn's disease. Gastroenterology. 2004;127(2):412-421.

41. Kim YG, Park JH, Shaw MH, Franchi L, Inohara N, Núñez G. The cytosolic sensors Nod1 and Nod2 are critical for bacterial recognition and host defense after exposure to Toll-like receptor ligands. Immunity. 2008;28(2):246-257.

42. Goodridge HS, Simmons RM, Underhill DM. Dectin-1 stimulation by Candida albicans yeast or zymosan triggers NFAT activation in macrophages and dendritic cells. JImmunol. 2007;178(5):3107-3115.

43. Cooney R, et al. NOD2 stimulation induces autophagy in dendritic cells influencing bacterial handling and antigen presentation. Nat Med. 2010;16(1):90-97.

44. Chuang LS, et al. A frameshift in CSF2RB pre- dominant among Ashkenazi Jews increases risk for Crohn's disease and reduces monocyte signaling via GM-CSF. Gastroenterology. 2016;151(4):710-723.e2.

45. Homer CR, Richmond AL, Rebert NA, Achkar JP, McDonald C. ATG16L1 and NOD2 interact in an autophagy-dependent antibacterial pathway implicated in Crohn's disease pathogenesis. Gastroenterology. 2010;139(5):1630-1641.e1.

46. Lassen KG, et al. Genetic coding variant in GPR65 alters lysosomal pH and links lysosomal dysfunction with colitis risk. Immunity. 2016;44(6):1392-1405.

47. Schwerd T, et al. Impaired antibacterial autophagy links granulomatous intestinal inflammation in Niemann-Pick disease type C1 XIAP deficiency with NOD2 variants in Crohn's disease [published online ahead of March 7, 2016]. Gut. https://doi.org/10.1136/gutjnl-2015-310382.

48. Uhlig HH, et al. The diagnostic approach to monogenic very early onset inflammatory bowel disease. Gastroenterology. 2014;147(5):990-1007.e3.

49. Rivas MA, et al. Deep resequencing of GWAS loci identifies independent rare variants associated with inflammatory bowel disease. Nat Genet . 2011;43(11):1066-1073.

50. Benzinger A, Muster N, Koch HB, Yates JR, Hermeking H. Targeted proteomic analysis of 14-3-3 sigma, a p53 effector commonly silenced in cancer. Mol Cell Proteomics. 2005;4(6):785-795.

51. Vincenz C, Dixit VM. 14-3-3 proteins associate with A2O in an isoform-specific manner and function both as chaperone and adapter molecules. J Biol Chem. 1996;271(33):20029-20034.

52. Liu Y, et al. Genome-wide interaction-based association analysis identified multiple new susceptibility loci for common diseases. PLoS Genet. 2011;7(3):e1001338.

53. Tampella G, et al. The Tec kinase-regulated phosphoproteome reveals a mechanism for the regulation of inhibitory signals in murine macrophages. JImmunol. 2015;195(1):246-256.

54. Morandi F, et al. Soluble HLA-G dampens CD94/ NKG2A expression and function and differentially modulates chemotaxis and cytokine and chemokine secretion in CD56 $6^{\text {bright }}$ and CD56 $6^{\text {dim }}$ NK cells. Blood. 2011;118(22):5840-5850.

55. Keerthivasan G, et al. Aberrant overexpression of CD14 on granulocytes sensitizes the innate immune response in mDia1 heterozygous del $(5 \mathrm{q})$ MDS. Blood. 2014;124(5):780-790.

56. Lee IH, et al. A role for the NAD-dependent deacetylase Sirt1 in the regulation of autophagy. Proc Natl Acad Sci U S A. 2008;105(9):3374-3379.

57. Musial A, Eissa NT. Inducible nitric-oxide synthase is regulated by the proteasome degradation pathway. J Biol Chem. 2001;276(26):24268-24273.

58. Gianni D, DerMardirossian C, Bokoch GM. Direct interaction between Tks proteins and the $\mathrm{N}$-terminal proline-rich region (PRR) of NoxA1 mediates Nox1-dependent ROS generation. Eur J Cell Biol. 2011;90(2-3):164-171.

59. Chen D, Hinkley CS, Henry RW, Huang S. TBP dynamics in living human cells: constitutive association of TBP with mitotic chromosomes. Mol Biol Cell. 2002;13(1):276-284. 